\title{
Anmerkungen zur Bauernpolitik der Deutschen Kommunistischen Partei
}

\section{Uif Baumgärtner}

Das Bild des Bauern als ,Kostgänger der Gesellschaft" und der Landwirtschaft als einer mit Subventionsgeldern aufrechterhaltenen Idylle, das von der bürgerlichen Presse gezeichnet wird, hat sicherlich auch auf die Haltung der Linken in der Bauernfrage abgefärbt. Hinzu kommt, daß es in der Arbeiterbewegung immer wieder Strömungen gab, die die Bauern rechts liegen lassen wollten. Das Guthaer Programm der Sozialdemokratischen Partei von 1875 formulierte, gegenuber der Arbeiterklasse seien ,alle anderen Klassen eine reaktionäre Masse“" und das Erfurter Programm von 1891 vertrat die These, daß alle Klassen außer der Arbeiterklasse ,die Erhaltung der Grundlagen der heutigen Gesellschaft zum gemeinsamen Ziel haben."(1) Bucharin stellte in einer Rede „Über die Bauernfrage" vor der Erweiterten Exekutive der Komintern 1925 fest, daß in den ,zentralen Ländern England, Deutschland und Amerika ... unser Einfluß auf die Bauernschaft gleich Null ist." (2) Daran dürfte sich bis zur Machtergreifung Hitlers nicht viel geändert haben, obwohl die KPD gleich im folgenden Jahr ,Das Gesicht dem Dorfe zu! " (3) -wandte und die Landrevue der DKP heute schreibt: „Kommunisten auf dem Land - Bauernpolitik hat große Tradition." (4) Die Tatsache, daß das Bauernhilfsprogramm der KPD erst 1931 verkündet wurde, spricht für sich.

Auch nach dem 2. Weltkrieg haben die Bauern lange Zeit in der Politik der bundesrepublikanischen Linken ein Schattendasein geführt. Erst als sie auf die Straße gingen, besonders bei den großen Demonstrationen vom Frühjahr 1971, rückten sie auf die Titelseiten der bürgerlichen Presse und ins Bewußtsein linker Gruppierungen. Zur selben Zeit hat auch die DKP die Arbeit unter den Bauern verstärkt in Angriff genommen: am 27./28. Februar 1971 wurde das „Demokratische Bauernprogramm der Deutschen Kommunistischen Partei ${ }^{66}$ vom Parteivorstand beschlossen (es versäumt nicht, sich auf die ,gewaltigen Demonstrationen“ der westdeutschen Bauern zu berufen) und ebenfalls im Frühjahr 1971 erschien die erste Nummer der „DKP-Landrevue. Informationen für die Landbevölkerung.“ Das Motiv für das Interesse der DKP an den Problemen der Bauern ergibt sich zunächst aus ihrer marxistischen Tradition: ,Als marxistische Partei erkennt sie die Bauernschaft als wichtigsten Verbündeten der Arbeiterklasse im Kampf gegen die Allmacht des Monopolkapitals ..." (5). Aber das Programm der DKP ist auch, ,in der Praxis geboren": „der Entwurf des,Demokratischen Bauernprogramms der DKP $^{6}$ ist fast zwei Jahre lang in der Öffentlichkeit zur Diskussion gestellt worden." (6) Ergebnis dieses den "demokratischen Grundsätzen unserer Partei

1) Programm der Deutschen Sozialdemokratie, Anhang zu Marx: Kritik des Gothaer Programms, Verlag Neuer Weg, Berlin 1946.

2) Nikolai Bucharin, Úber die Bauernfrage, Verlag Karl Hoym Nachf, Hamburg 1925.

3) Ernst Thälmann, Das Gesicht dem Dorfe zu!, Rote Fahne, Berlin, 14. 2. 19.26.

4) DKP-Landrevue. Jahrgang 1, Nr. 3/1971, S. 14.

5) Demokratisches Bauernprogramm der DKP, Düsseldorf 1971, S. 7.

6) DKP-Landrevue, Jahrgang 1, Nr. 2/1971, S. 3. 
entsprechenden " Verfahrens: Umstellung einiger Passagen und minimale Veränderungen in der Formulierung.

Eine praktische Kritik der DKP-Bauernpolitik muß in erster Linie mit einer Kritik der Praxis beginnen. Aber auch nach der Entschließung des Düsseldorfer Parteitages „Zur Arbeit der Partei auf dem Lande“, mit der die Mitglieder zu verstärkter Öffentlichkeitsarbeit auf dem Lande verpflichtet werden, hat es an Gelegenheit gefehlt, die praktische Tagesarbeit der DKP in den Dörfern zu beobachten. Von Zeit zu Zeit wird von einem „Agitationseinsatz" (7) berichtet, über Dorfversammlungen und das Verteilen der DKP-Landrevue (8) oder über Fahrten westdeutscher Bauern in die DDR zur Besichtigung von LPG's (9). Natürlich hat die DKP-Presse auch ausführlich über den Milchkrieg in Weiding berichtet, bei dem die Vertragsbauern der Allgäuer Alpenmilch AG für höhere Auszahlungspreise kämpften. Jedoch zeigt gerade dieses Beispiel, worauf an anderer Stelle noch näher eingegangen wird,

- wie die DKP am Rande stehen blieb und sich nicht in diese Bewegung einschalten konnte,

- wie sie deshalb zu einer falschen Interpretation des Milchkrieges als einer eindeutig antimonopolistischen Aktion kam.

Es ist die mangelnde Möglichkeit einer praktischen Auseinandersetzung mit der DKP-Bauernpolitik, die uns vorläufig auf eine Einschätzung ihrer theoretischen Grundlagen und programmatischen Äßerungen beschränkt. Die eigentliche Absicht der folgenden Überlegungen bleibt aber eine auf die Praxis bezogene Auseinandersetzung. Denn uns treibt nicht die Vorstellung, es gehe um eine ,antirevisionistische Entlarvung" der DKP an jedem Ort und bei jedem Problem. Vielmehr ist der Ausgangspunkt unserer Kritik unsere eigene Erfahrung in der politischen Arbeit mit Bauern. Dabei hat sich uns (der Verfasser spricht also als Vertreter einer Gruppe) die kritische Auseinandersetzung mit der letzten Endes unbestimmten und aussichtslosen, ja sogar verwirrenden Bauernpolitik der DKP aufgedrängt.

Schon eine oberflächliche Lektüre des Bauernprogramms der DKP und der bisher erschienenen Ausgaben der Landrevue bestätigt, was zu vermuten war: die DKP verlängert den Kampf aller demokratischen Kräfte für die antimonopolistische Demokratie geradlinig aufs Land. Dennoch erfordert eine gründlichere Auseinandersetzung mit diesem Teil der DKP.Politik ein längeres Verweilen bei den einzelnen Punkten unter Zuhilfenahme weiterer Quellen, insbesondere des Buches „Arbeiter und Bauern gegen Bosse und Banken" (10), das als analytisches Fundament für die DKP-Bauernpolitik betrachtet werden kann. Es zeigt sich, daß die Klassenanalyse auf dem Lande der Theorie vom staatsmonopolistischen Kapitalismus angepaßt wird.

7) Nahrungsmittelkonzerne treiben die Preise hoch. Gespräch mit Bauern im Raum Göttingen. Unsere Zeit (UZ), 1. 5. 1973.

8) DKP-Landrevue, Jahrgang 2, Nr. 3/1972, S. 13.

9) DKP-Landrevue, Jahrgang 1, Nr. $3 / 71$.

10) Richard Scheringer und Wilhelm Sprenger, Arbeiter und Bauern gegen Bosse und Banken. Verlag Marxistische Blätter, Frankfurt a. M., 1970. 
Deshalb zunächst eine Auseinandersetzung mit der DKP-Einschätzung der Klassenstruktur in der westdeutschen Landwirtschaft, ihrer Unterordnung unter die Stamokap-Theorie und der aus beidem resultierenden Neuformulierung der Bündnispolitik.

\section{Die Klassen in der Landwirtschaft der BRD}

Das Demokratische Bauernprogramm umgeht die Frage der Klassenstruktur auf dem Lande. In ihm ist lediglich von "Bauern", "werktätigen Bauern" und „,moderner bäuerlicher Landwirtschaft" die Rede. Scheringer/Sprenger nennen als Faktoren der Klassenstruktur „Bodenfläche, Beschäftigung von Lohnarbeitern, Viehbesatz, Maschinenausrüstung, Einkommensverhältnisse u. a." (11), verwenden dann aber vor allem die Betriebsgröße als Klassenkriterium. Dabei stellen sie fest, daß sich die „Klassengrenzen innerhalb der Bauernschaft nach oben" (12) verschoben haben. Ein Vergleich zwischen 1949 und 1969 ergibt (13):

1949

1969

Halbproletarier

Kleinbauern

Mittelbauern

Großbauern

Kapitalistische Großbetriebe

$$
\begin{array}{cr}
0,5-2 \text { ha LN } & 0,5-5 \text { ha LN } \\
2-5 \text { ha LN } & 5-15 \text { ha LN } \\
5-20 \text { ha LN } & 15-50 \text { ha LN } \\
20-100 \text { ha LN } & 50-100 \text { ha LN } \\
100 \text { ha LN und } & 100 \text { ha LN und } \\
\text { mehr } & \text { mehr }
\end{array}
$$

Hinzu kommen noch die Landarbeiter und die Großgrundbesitzer, für die an anderer Stelle ebenfalls eine Betriebsfläche von über 100 ha $\mathrm{LN}$ angegeben wird (14).

Heinz Jung, der sich hauptsächlich auf die Arbeit von Scheringer/Sprenger stützt, meint einerseits, daß sich auch im Agrarsektor „die Anwendung von Lohnarbeit" als wesentliche Klassendifferenzierungslinie ergibt, fährt dann aber fort: „Als grober Anhaltspunkt, in dem unter gegebenen Verhältnissen die übrigen Kriterien mehr oder weniger zum Ausdruck kommen, kann jedoch der Umfang der LN benutzt werden." (15)

Die Klassengliederung nach Betriebsgrößen ist bestenfalls ungenau, oft aber falsch. So waren z. B. bereits 1960 in den Betrieben zwischen 20 und 50 ha nur

11) Scheringer/Sprenger, a. a. O., S. 66.

12) Ebenda, S. 76.

13) Ebenda, S. 174, Tab. 7. LN: landwirtschaftliche Nutzfläche.

14) Scheringer/Sprenger, a. a. O., S. 67.

15) Heinz Jung, Zu den klassentheoretischen Grundlagen einer sozialstatistischen Analyse der Klassen- und Sozialstruktúr der BRD. In: Klassen- und Sozialstruktur der BRD 1950-1970. Theorie, Diskussion, Sozialstatistische Analyse. Teil I: Klassenstruktur und Klassentheorie. Theoret. Grundlagen und Diskussion. Institut für marxistische Studien und Forschungen (IMSF), Frankfurt a. M. 1972, S. 149, 151. 
noch $87,6 \%$ der Betriebsinhaber voll im land- und forsíwirtschaftlichen Betrieb beschäftigt (16). Mit anderen Worten: $12,4 \%$ der von Scheringer/Sprenger als Mittelbauern klassifizierten waren auf dem Weg zum Zum oder Nebenerwerb, d. h. auf dem Weg ins Halbproletariat. Nach der Landwirtschaftszählung von 1960 bezogen damals $42,3 \%$ aller Betriebsinhaber den überwiegenden Teil ihres Lebensunterhaltes aus nicht landwirtschaftlichen Quellen. In den Betrieben unter 5 ha waren es $73,4 \%$ und in den Betrieben zwischen 5 und 10 ha (die nach Scheringer/Sprenger zu den Kleinbauernbetrieben zählen) waren es irmmerhin noch $15 \%$ (17). Unberïcksichtigt bleibt bei einer solchen, "Klassengliederung" auch der landwirtschaftliche Standort: 5 ha Gemüse oder Wein reichen für eine mittelbäuerliche Existenz aus, 5 ha Getreide nicht.

Auch das Kriterium der Beschäftigung von Lohnarbeit befriedigt nicht. Zwar kann man in Anlehnung an Lenin mit Scheringer/Sprenger sagen, daß, ,in einigen mittelbäuerlichen Betrieben, oft nur zeitweise, fremde Arbeitskräite ausgebeutet

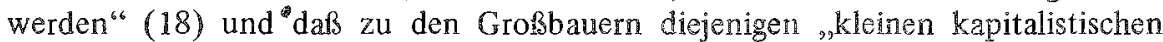
Unternehmen in der Landwirtschaft ${ }^{60}$ zu zählen sind, die ,einige Lohnarbeiter ausbeuten (19). Aber aufgnund der Tatsache, daß nur noch $10 \%$ aller in der Landwirtschaft beschäftigten Arbeitskräfte Lohnarbeiter sind und daß nur noch in $7,7 \%$ der Betriebe Lohnarbeiter beschäftigt werden, würde, allein auf dieses Kriterium gestützt, einem geringen Prozentsatz eindeutig als kapitalistisch zu definierender landwirtschaftlicher Betriebe die große ungegliederte Masse der übrigen Betriebe gegenüberstehen. Entgegen Lenins Ansicht, daß ,das unbestreitbarste und direkteste Kennzeichen für die Entwicklung des Kapitalismus (in der Landwirtschaft - d. V.) natülich der Grad der Verwendung von Lohnarbeit ist " (20), reicht dieses Kriterium angesichts der kapitalistischen Durchdringung der Landwirtschaft in Form der vertikalen Integration (Vertragslandwirtschaft) für die gründliche Untersuchung der sozialen Verhältnisse auf dern $\mathbb{L}$ ande nicht mehr aus. Rechtziegler schreibt in diesem Zusammenhang: es reicht ,bei weitem nicht mehr aus, wenn es gilt, die soziale Gliederung der bäuerlichen Bevölkerung zu ermitteln, mit Zahlen über familieneigene und familienfremde Arbeitskräfte $z u$ operieren." $(21)$

So unbefriedigend das Kriterium der Anwendung von Lohnarbeit in der Landwirtschaft für die Erarbeitung einer differenzierten Strategie sozialistischer Bauernpolitik ist, so muß bei der weiteren Untergliederung derjenigen bäuerlichen Produzenten, die nicht eindeutig als Kapitalisten anzusprechen sind, doch klar unterschieden werden zwischen dem Produktionsverhältnis, d. h. dem Verhältnis zu Lohnarbeit und Kapital, und sozioökonomischen Charakteristika, mit deren Hilfe

16) Emil Rechtziegler, Neue Entwicklungstendenzen in der Landwirtschaft der kapitalistischen Staaten. VEB Deutscher Landwirtschaftsverlag, Berlin 1968, S. 151.

17) Rechtziegler, Neue Entwicklungstendenzen ..., a. a. O., S. 137

18) Scheringer/Sprenger, a. a. O., S. 157.

19) Scheringer/Sprenger, a. a. O., S. 158.

20) W. I. Lenin, Neue Daten über die Entwicklungsgesetze des Kapitalismus in der Landwirtschaft, in: Werke Bd. 22, Berlin 1960, S. 38.

21) Rechtziegler, Neue Entwicklungstendenzen ..., a. a. O., S. 103. 
die Differenzierungsprozesse innerhalb der keine Lohnarbeit ausbeutenden Landwirtschaft und daraus resultierende Veränderungen im Bewußtsein und politischen Verhalten erfaßt werden können. Diese beiden Ebenen werden bei Scheringer/ Sprenger vermischt und auch Jung zählt ohne Unterscheidung „Umfang der landwirtschaftlichen Nutzfläche, Anwendung von Lohnarbeit, Maschinenausrüstung, Einkommensverhältnisse u. a." zu den „empirischen Kriterien einer eingehenden Analyse. ${ }^{6}$ (22)

Scheringer/Sprenger benutzen denn auch die Fähigkeit eines landwirtschaftlichen Betriebes zur erweiterten Reproduktion als Klassenkriterium. Danach erlauben günstige Umstände (gute Ernten in manchen Jahren usw.) den Mittelbauern ,eine bescheidene erweiterte Reproduktion" (23), während die Großbauern in der Lage sind, ,moderne Produktionsmittel anzuwenden und das Produktionsniveau zu steigern" (24), also erweitert zu reproduzieren. Bedenkt man, daß die erweiterte Reproduktion ohne Anwendung von Lohnarbeit (im sogenannten Einmannbetrieb) ein wesentliches Ergebnis der Entwicklung der landwirtschaftlichen Produktivkräfte unter kapitalistischen Bedingungen war und ist, dann erscheint die Koppelung von erweiterter Reproduktion und Ausbeutung "on Lohnarbeitern, die Scheringer/Sprenger bei der Beschreibung der Großbauern vornehmen, klassenanalytisch unhaltbar. Bei einer solchen Verwendung sozioökonomischer Daten entsteht dann nämlich der Eindruck, als ob erweiterte Reproduktion mit kapitalistischer Produktion und einfache Reproduktion mit einfacher Warenproduktion gleichzusetzen wären. Ebensowenig wie eine bestimmte Betriebsgröße repräsentiert ein bestimmter Besatz mit Produktionsmitteln notwendigerweise ein bestimmtes Produktionsverhältnis.

Wenn Lenin, dessen methodisches Vorgehen Scheringer/Sprenger in diesem Punkt wohl nachahmen, in seiner Arbeit ,Andie Dorfarmut ${ }^{66}$ (25) ebenfalls die Ausstattung mit Produktionsmitteln zur Beschreibung der russischen Agrarverhältnisse benutzt hat, indem er zwischen pferdelosen Bauern (= Dorfarmut), die völlig mittellos und zum Proletariat zu zählen sind, Bauern mit nur einem Pferd (Halbproletarier) und Bauern mit einem Gespann Zugvieh (Mittelbauern) unterscheidet, dann ist zu bedenken,

daß im zaristischen Rußland der soziale Unterschied zwischen einem auf der Handarbeitsstufe arbeitenden Bauern und einem mit tierischer Zugkraft arbeitenden ungleich größer war, als der Unterschied zwischen einem Bauern mit einem 20-PS-Schlepper und einem mit einem 50-PS-Schlepper bei uns heute;

daß die nussische Dorfarmut in einem feudalen Abhängigkeitsverhältnis zu den Großgrundbesitzern stand, eine eindeutige Beziehung zwischen sozioökonomischer- und Klassenlage also gegeben war, während die Bauern in der Bundesrepublik, die zu keiner erweiterten Reproduktion fähig sind, ebenso

22) Jung, a. a. U., S. 149.

23) Scheringer/Sprenger, a. a. O., S. 157.

24) Ebenda, S. 158.

25) W. I. Lenin, Werke Bd. 6, S. 382-386. 
gut einfache Warenproduzenten wie Arbeiter-Bauern sein können.

Ahnlich ist die Heranziehung des Verschuldungsgrades als Klassenkriterium zu beurteilen. Auch er gibt keine Auskunft darüber, in welchem Produktionsverhältnis ein Baver produziert.

Die allgemein gebräuchlichen Begriffe Klein-, Mittel- und Großbauern oder auch arme und reiche Bauern beschreiben also eher quantitative Unterschiede in der Wirtschaftskraft als qualitativ verschiedene Produktionsverhältnisse; ,die Aufmerksamkeit (wird) viel eher auf relative Unterschiede des Reichtums ..., die man nur willkürlich in Kategorien verwandeln kann, (konzentriert), als auf Klassenbeziehungen, die klar definiert werden müssen." $(26)$

Tatsächlich hat gerade der Begriff Mittelbauern zu formallogischen Kopfsprüngen Anlaß gegeben. So schreibt z. B. N. G. Karotamm: „Der Mittelbauer steht in der Mitte zwischen dem Reichen und dem Proletarier - darum heißt er auch Mittelbauer.“(27) Ebenso nichtssagend ist es, wenn Jung die Mittelbauern als Oberschicht der Kleinbauern bezeichnet (28).

Die bisher genannten Kriterien sind wohl auf die gegenwärtige Situation in der BRD-Landwirtschaft anwendbar, aber sie tragen den neuen Entwicklungen, dem gegenwärtigen Vordringen des Kapitals in die Landwirtschaft, das tendenziell auf die Liquidation der Bauern als kleine Warenproduzenten zielt (allerdings werden vermutlich noch sehr lange Sektoren mit einfacher Warenproduktion überleben, deren Übernahme sich für das Kapital nicht lohnt), noch nicht Rechnung. Seit das Kapital begonnen hat, den Feudalismus auch aus der Landwirtschaft zu vertreiben, findet ein unaufhörlieher Differenzierungsprozeß innerhalb der Bauernschaft statt. Die Haupttendenz dabei ist die bereits im ,Manifest der Kommunistischen Partei" beschriebene: „Die ganze Gesellschaft spaltet sich mehr und mehr in zwei große feindliche Lager, in zwei große, einander direkt gegenüberstehende Klassen: Bourgeoisie und Proletariat." (29) Die Form und das Ausmaß, in denen diese Differenzierung auf dem Lande stattfindet, hängen vom jeweiligen Entwicklungsstand der kapitalistischen Gesellschaft $a b$, von den historischen Umständen. Trotzky meinte für das revolutionäre Rußland: „Für mich beginnt die Frage mit der Negation der Bauernschaft als eines Ganzen. Es handelt sich um den Klassenkampf innerhalb der Bauernschaft. Die mittleren Bauern stellen ein ganz und gar besonderes Protoplasma dar. Die Ausformung dieses Protoplasmas vollendet sich unvermeidlich in zwei Richtungen: in der kapitalistischen über die Kulaken, in der sozialistischen über die halbproletarischen Bauern und Landarbeiter." (30)

Dieser Übergangsprozeß nach den beiden Hauptklassen der kapitaistischen Gesellschaft hin wird von Scheringer/Sprenger nur einseitig erfaßt, wenn sie davon

26) Hamza Alavi, Theorie der Bauernrevolution, Plakat-Bauernverlag, Stuttgart 1972, S. 7.

27) N. G. Karotamm, Geschichtliches zur Lehre von der sozialistischen Landwirtschaft. Dietz Verlag, Berlin 1962, S. 59.

28) Jung, a. a. O., S. 148.

29) Marx/Engels, Manifest der Kommunistischen Partei. In: Marx/Engels, Werke Bd. 4, Dietz Verlag, Berlin 1971, S. 463.

30) Brief Trotzkis an Boris Souvarine vom 23. April 1929. In: Boris Souvarine: Contribution à l'histoire du Comintern. Librairte Droz, Genf 1965. 
ausgehen, daß ,für alle bäuerlichen Schichten ein allgemeiner sozialer Abstieg symptomatisch (ist)" (31). Dies ist der einzige Punkt, an dem Jung die Arbeit von Scheringer/Sprenger kritisiert: „Es muß aber gerade bei der Analyse dieser Gruppen den Übergangsmöglichkeiten in andere Gruppen ländlicher Mittelschichten und in neue Gruppen der ländlichen Bourgeoisie Aufmerksamkeit gewidmet werden, wenn die Differenzierungsprozesse nicht einseitig erfaßt werden sollen."(32) Gleichwohl beschränkt er sich darauf, eine daraus resultierende Widersprüchlichkeit anzumerken, ,die in der praktischen Einbeziehung der Bauernschaft in antimonopolistische Bündnisse wirksam ist" (33) - ohne die Übergangsmöglichkeiten nach „oben" näher zu untersuchen, ohne die Schwierigkeiten für das Arbeiter-BauernBündnis zu benenıen. Es offenbart sich hier die Tendenz zum „Primat der Taktik über die Theorie" (34); dazu, die Analyse den taktischen Wunschvorstellungen unterzuordnen.

Wie sieht der Differenzierungsprozeß, der sich aus dem Widerspruch zwischen den wachsenden Produktivkräften und der Agrarstruktur der Bundesrepublik entwickelt, gegenwärtig aus? Scheringer/Sprenger stellen vier Tendenzen fest: (35)

- Zunahme der Betriebe über 20 ha. Die Bedeutung dieses „klassischen“ Konzentrationsprozesses geht zurïck.

- Horizontale monopolkapitalistische Kooperation, „Zusammenschluß ehemals bäuerlicher Betriebe unter dem Kommando von Kapitalisten und Monopolen".

- Vertikale monopolkapitalistische Kooperation, „Organisation des Produktions- und Reproduktionsprozesses in der Nahrungsgüterindustrie, bei der die Industrie- und Handelsmonopole in die landwirtschaftliche Produktion eindringen".

- Errichtung gewerblicher Großbetriebe durch die Monopole, vor allem in der bodenunabhängigen Produktion.

Neben der Ruinierung der Kleinbauern durch Übergang zum Nebenerwerb und schließlich zur gänzlichen Betriebsaufgabe streichen Scheringer/Sprenger vor allem die Tendenz heraus, daß Mittel- und sogar Großbauern zu „De-Facto-Lohnarbeitern“ absteigen (36), daß der landwirtschaftliche Betrieb „,eine abhängige Betriebseinheit innerhalb eines größeren Unternehmens"(37) wird. Rechtziegler schreibt: „Es besteht die Tendenz, daß sich mit der vertikalen Integration der landwirtschaftliche Produzent schließlich in einen ,besitzenden" Lohnarbeiter verwandelt.“(38) Und: „Bei straffen Formen der vertikalen monopolkapita-

31) Scheringer/Sprenger, a. a. O., S. 66.

32) Jung, a. a. O., S. 152.

33) Ebenda.

34) Korte, Nolle, Witt, Wölk, Staat und SPD im Monopolkapitalismus (Zur Kritik der Theorie des Stamokap), SOAK, (Sozialist. Aktion. Materialien zur sozialistischen Theorie und Praxis), März 1973, S. 4.

35) Scheringer/Sprenger, a. a. O., S. $83 \mathrm{f}$.

36) Ebenda, S. 95.

37) K. Kroeschell, Der Landwirt als Unternehmer - ein neues Rechtsproblem. Zitiert bei Scheringer/Sprenger, a. a. O., S. 93, 94.

38) Rechtziegler, Neue Entwicklungstendenzen ..., a. a. O., S. 182. 
listischen Kooperation tritt der Marktpreis ganz in den Hintergrund. Seine Stelle nimmt ein ,Verrechnungspreis“ ein, der unabhängig vom Markpreis gebildet wird. " (39) Rechtziegler zitiert auch Jäger, der von „Pseudo-Bauern, weisungsgebundenen Bewirtschaftern und landwirtschaftlichen Heimarbeitern ${ }^{65}$ spricht (40). Denselben Ausdruck gebraucht Lambert, wenn er von der Vertragslandwirtschaft spricht: „Somit wird der Bauer zum Heimarbeiter, zum totalen Gefangenen, gefesselt durch seine Verschuldung. ${ }^{\text {" }}$ (41)

Für die Gliederung der Klassen auf dem Lande und eine daran auszurichtende sozialistische Politik ist es wesentlich, nicht nur die oben beschriebenen Tendenzen aufzuzeigen, sondern die gegenwärtig auftretenden Übergangsklassen des Differenzierungsprozesses zu fassen. Lambert tut das, indem er die Kategorien pauperisierte und proletarisierte Bauern einführt. Er definiert sie folgendermaßen:

„Pauperisierte oder arme Bauern sind solche, die in kleinen Betrieben arbeiten und nicht das Risiko auf sich genommen haben, sich in Investitionen zu stürzen, die die Industrialisierung der Produktion ermöglichen. Sie werden sowohl von den Großbauern ausgebeutet, die sich ihr Elend zunutze machen, als auch von den Behörden, die zugunsten des Kapitalismus ihre Abwanderung beschleunigen, indem sie ihre Einkünfte mehr und mehr einschränken. So werden sie zu Entwurzelten, die für eine Handlangerfunktion in der Industrie bereitstehen, zu welchem Lohn auch immer.

„Proletarisierte Bauern sind solche, die sich auf eine intensive Modernisierung, die Industrialisierung ihrer Produktionsmittel eingelassen haben. Sie sind hochverschuldet urd werden vollständig von den Industrien und Genossenschaften beherrscht, die ihnen die Ausstattungen liefern und für Absatzgebiete sorgen. Als Arbeiter ausgebeutet, werden sie langsam der wirklichen Produktionsmittel enteignet. So gelangen sie nach und nach in die Kategorie der Proletarier." (42)

Für die erste Kategorie ergibt sich also als Klassenkriterium der Grad, mit dem ein Bauer seinen Lebensunterhalt aus nicht landwirtschaftlichen Quellen deckt, wobei es für eine weitere Untergliederung natürlich entscheidend ist, ob diese Quelle in Holzverkäufen oder Krediten besteht, oder in der industriellen Lohnarbeit von

39) Rechtziegler, Westdeutsche Landwirtschaft im Spätkapitalismus. DWI-Forschungshefte, 4. Jahrgang, Heft 4, 1969.

40) Rechtziegler, Neue Entwicklungstendenzen ..., a. a. O., S. 182.

41) Bernard Lambert, Bauern im Klassenkampf. Rotbuch 32, Verlag Klaus Wagenbach, Berlin 1971, S. 44.

42) Ebenda, S. 48. - Lambert erläutert nicht näher, wie sich die Großbauern das Elend der pauperisierten Bauern zunutze machen. Dies geschieht wohl vor allem über die politische Vormachtstellung der Großbauern in den Genossenschaften und berufsständigen Organisationen.

Die beiden von Lambert benutzten Kategorien entsprechen den beiden Haupttendenzen im Ruinierungsprozeß der Bauern: dem traditionellen Übergang vom Mittel- urid Kleinbauern zum Arbeiter-Bauern einerseits und der neuen Form der Vertragslandwirtschaft andererseits. Die in der Übersetzung benutzten Partizipien geben sprachlich den Prozeßcharakter nicht wider; es wäre besser von Bauern, die pauperisiert werden und Bauern, die proletarisiert werden, zu sprechen.

Der Ausdruck Pauper charakterisiert überdies jenen Teil der industriellen Reservearmee auf dem Lande, der bewußtseinsmäßig am wenigsten mobil ist. 
Familienmitgliedern oder des Betriebsinhabers selbst. Für die zweite Kategorie muß der Grad der wirtschaftlichen Abhängigkeit von den Konzernen im Rahmen der Vertragslandwirtschaft trotz formeller Aufrechterhaltung des bäuerlichen Eigentums als Klassenkriterium verwandt werden. Sie nimmt von der einfachen Absatzvereinbarung über die Produktions- und Liefervereinbarung zur Vereinbarung über den Bezug von Produktionsmitteln zu. Bei den Lohnmastverträgen in der Schweinemast beispielsweise liefert der ,Integrator ${ }^{66}$ Ferkel und Futtermittel und bleibt während der Mastzeit Eigentïmer der Schweine und Futtermittel, während der Mäster seine Arbeitskraft, Stallräume $u_{0} a_{0}$ in den Vertrag einbringt. Der Bauer produziert hier also schon teilweise mit fremden Produktionsmitteln.

Die DKP - in diesem Fall ihre Agrarexperten Scheringer und Sprenger unternimmt im Unterschied zu Lambert nicht den Versuch, die Übergangsklassen des Ruinierungs- und Differenzierungsprozesses auf dem Lande zu analysieren. Der Grund dafür ist, daß sie sämtliche bäuerlichen Klassen und ihre Verschiebung im Verlaufe der kapitalistischen Durchdringung der Landwirtschaft der Haupttendenz unterordnet: „Unter Ruinierung der Bauern verstehen wir ihre Verwandlung aus einfachen Warenproduzenten in Lohnarbeiter ${ }^{66}(43)$. Es kommt also zu einer "Annäherung der verschiedenen Schichten innerhalb der Bauernschaft" (44). Rechtziegler betont in ähnlicher Weise die Haupttendenz: „Es kann gegenwärtig beobachtet werden, daßs sich in Westdeutschland vor allem eine breite Differenzierung nach unten durchsetzt ${ }^{64}(45)$. Was in der Tendenz zweifellos stimmt, wird in der praktischen Bauernpolitik der DKP de facto als abgeschlossener Prozeß genommen, wenn sich das Demokratsiche Bauernprogramm pauschal an die „Bauernschaft" wendet. Wo, wie in dem Buch von Scheringer/Sprenger, überhaupt noch klassenmäßig argumentiert wird, werden die Leninschen Klassenkriterien übernommen, ohne sie weiter zu entwickeln. Dabei ist in der obersten Kategorie, derjenigen der ,agrarkapitalistischen Betriebe", eine gewisse Begriffsverwirrung festzustellen (46).

43) Scheringer/Sprenger, a. a. O., S. 69.

44) Ebenda, S. 160.

45) Rechtziegler, Neue Entwicklungstendenzen ..., a. a. 0., S. 162.

46) Das Demokratische Bauernprogramm spricht abwechselnd von ,kapitalistischen Agrarunternehmern" (a. a. O., S. 18), ,Großgrundeigentum" und ,feudalem und großkapitalistischem Grundbesitz" (S. 18). Scheringer/Sprenger benutzen eine Klassengliederung, bei der "Großbauern" und "Großgrundbesitzer" als der "Klasse der Kapitalisten" zugehö:; erscheinen (S. 67). An anderer Stelle sprechen sie von ,kapitalistischen Großbetrieben" und definieren sie als jene 2800 Betriebe, die über 100 ha LN besitzen. (S. 79) Daneben werden auch die Großgrundbesitzer beschrieben (S. 80), wobei die ,fuirstliche Domänenverwaltung" der Thurn und Taxis beispielsweise als, ,Monopolunternehmen" bezeichnet wird. Demnach besteht zwischen "Großbauern", die einige Lohnarbeiter ausbeuten (Scheringer/Sprenger, S. 158), ,kapitalistischen Großbetrieben ${ }^{6}$ und dem wie ein Monopolunternehmen organisierten ,Großgrundbesitz" kein definierter Unterschied.

Den in der BRD noch existierenden Großgrundbesitz als feudal zu bezeichnen, ist unzulässig, denn erstens ist dieser Großgrundbesitz voll in den kapitalistischen Mark integriert und zweitens basiert er auf einer Lohnarbeitsverfassung und nicht auf einer feudalen Arbeitsverfassung mit Hörigen oder Leibeigenen. (Ein feudaler Überrest besteht 
Die Untersuchungen der DKP über die Klassenstrukturen in der westdeutschen Landwirtschaft weisen zwei grundlegende Mängel auf:

- Es wird keine Unterscheidung getroffen zwischen der Bestimmung der Klassenzugehörigkeit und der Beschreibung sozioökonomischer Verhältnisse. Die aus dem Vordringen des Kapitals in die Agrarproduktion sich ergebende Differenzierung innerhalb der Bauernschaft wird einseitig als Verschiebung der „Sozialstruktur der Landwirtschaft nach ,links" “(47) gesehen, offensichtlich aus dem Wunsch heraus, eine „Dominanz antimonopolistischer Interessen ${ }^{66}(48)$ konstatieren zu können.

Demgegenüber lassen sich, wenn man bei der Bestimmung der Klassenzugehörigkeit von Produktionsverhältnissen ausgeht, innerhalb der Bauernschaft der BRD folgende Gruppen unterscheiden:

Kapitalistische Bauern, die Lohnarbeiter ausbeuten.

Selbstständige Warenproduzenten mit eigenen Produktionsmitteln, ohne Ausbeutung von Lohnarbeit.

- Arbeiter-Bauern, die auf dem landwirtschaftlichen Betrieb über eigene Produktionsmittel verfügen, einen Teil ihrer Arbeitskraft aber an einen Kapitalisten verkaufen.

- Heimarbeiter im Rahmen der vertikalen Integration, die mit eigenen und fremden Produktionsmitteln arbeiten und je nach Vertragsform mehr oder weniger abhängig vom Integrator sind.

allenfalls in einer gewissen persönlichen Abhängigkeit, die aber politisch vermittelt wird, beispielsweise über die dominierende Stellung der Großgrundbesitzer in den politischen Institutionen der betreffenden Gemeinden.)

Interessant ist, daß das Demokratische Bauernprogramm eine Tendenz zur Landkonzentration feststellt $($, Vorgesehen ist, daß in der Bundesrepublik mindestens zwei Drittel der bäuerlichen Betriebe verschwinden - ihr Boden soll wenigen Großunternehmen zur Verfügung gestellt werden... S. 10), während Scheringer/Sprenger die Meinung vertreten, daß "trotz der Ruinierung hunderttausender Kleinbauern nicht von einer entsprechenden Konzentration der Fläche und der Produktion in Großbetrieben gesprochen werden kann" (S. 81). In der Tat haben wir bereits gesehen, daß die Zahl der Landarbeiter (Kriterium für einen kapitalistischen Landwirtschaftsbetrieb) abnimmt. Ebenso hat die Zahl der Betriebe über 100 ha abgenommen (1968 gegenüber 1949 um $6,6 \%$ ). Scheringer/Sprenger geben keine Auskunft darüber, ob das auf eine Konzentration in dieser Betriebsgrößenklasse zurückzuführen ist. Wichtiger ist die von Rechtziegler zitierte Feststellung, daß der Nestlé-Konzern z. B., um die von ihm insgesamt verarbeitete Milch auf eigenen Weiden zu erzeugen, eine Fläche ungefähr so groß wie die Schweiz benötigen würde. (K. Pritzkoleit, Monopole in der Nahrungsmittelindustrie. In: Agri-forum, München, Heft 5/1964, S. 32) Das bedeutet, daß die Degradierung der Bauern zu Heimarbeitern viel vorteilhafter für die Monopole ist, als die vom Demokratischen Bauernptogramm suggerierte direkte Übernahme der Bodenproduktion. Es macht auch deutlich, weshalb der Ausdruck, ,bäuerlicher Heimarbeiter" genauer ist als ,De-Facto-Lohnarbeiter".

Jung, a. a. O., S. 151 .

(8) Ebenda, S. 152 . 
Die Anwendung der Theorie vom staatsmonopolistischen Kapitalismus auf die Landwirtschaft in der $B R D$

Im Demokratischen Bauernprogramm wird der staatsmonopolistische Kapitalismus (Stamokap) nicht beim Namen genannt, aber im Abschnitt ,Die Bauern und die EWG" wird beschrieben, wie man sich die Zusammenarbeit von Staat und Monopolen vorstellt: „Die bisherige EWG- und NATO-Politik hat den Bauern und den Verbrauchern in der Bundesrepublik schweren Schaden zugefügt. Im Interesse der Vormachtbestrebungen des Finanzkapitals und seiner aggressiven Expansionspolitik wurden die Bauern zu Hunderttausenden geopfert. “(49) Popularisiert heißt es in einer Schlagzeile der DKP-Landrevue: „Was die Monopole und ihre Parteien mit den Bauern machen, ist eine Riesen-Schweinerei!" (50)

Im Bauernprogramm wird also die Veränderung der Agrarstruktur mit der außerökonomischen Macht der Monopole erklärt und völlig auf eine Analyse verzichtet, die von der Entfaltung der ökonomischen Gesetze des Kapitals und dem Wirksamwerden spezifischer Verwertungszwänge ausgeht.

Scheringer/Sprenger erläutern den Stamokap: „Das wichtigste Merkmal des staatsmonopolistischen Kapitalismus besteht in der immer engeren Verflechtung zwischen der ökonomischen Macht der Monopole und den Machtorganen des imperialistischen Staates sowie den Führungsorganen der herrschenden Parteien, gesellschaftlichen Organisationen und Institutionen."(51) Noch besser drückt Rechtziegler den Kern der Theorie vom Stamokap aus: „Unter den Bedingungen der Verschmelzung der Macht der Monopole mit der des Staates und der vollen Herausbildung des staatsmonopolistischen Herrschaftssystems verwandelt sich die Konzentration der ökonomischen Macht in den Händen des Finanzkapitals in politische Macht, die sich in staatlichen Maßnahmen zur staatsmonopolistischen Monopolisierung der Existenzbedingungen der Landwirtschaft und in der Schaffung eines staatlichen Ausbeutungssystems niederschlägt. Der Staat setzt die Rahmenbedingungen der monopolkapitalistischen Ausbeutung auch im Bereich der Landwirtschaft." (52) (Hervorhebung v. V.) Ungeklärt bleibt allerdings, ob Staat und Monopole tatsächlich verschmolzen sind oder ob der Staat nur die Rahmenbedingungen der monopolkapitalistischen Ausbeutung setzt. Im ersten Fall wird die dem Kapitalismus eigene Trennung von Wirtschaft und Politik, die nur widersprüchlich und begrenzt aufgehoben werden kann, als endguiltig überwunden angenommen (53). Im anderen Fall wird an der Definition des kapitalistischen Staates im Kommunistischen Manifest festgehalten: „Die moderne Staatsgewalt ist

49) Demokratisches Rauernprogramm der DKP, a. a. O., S. 24.

50) DKP-Land revue, Jahrgang 1, Nr. 4/71.

51) Scheringer/Sprenger, a. a. O., S. 119.

52) Rechtziegler, Westdeutsche Landwirtschaft ..., a. a. O., S. 81.

53) Margaret Wirth macht auf die Analogie dieser Vorstellung von einem geschlossenen, in: sich widerspruchslosen Machtapparat von Staat und Monopolen zum Kautskysche: Konzept des „Ultraimperialismus" aufmerksam. (Margaret Wirth, Kapitalismusthene. der DDR. edition suhrkamp, Nr. 562, Frankfurt/M. 1972, S. 69). 
nur ein Ausschuß, der die gemeinschaftlichen Geschäfte der ganzen Bourgeoisieklasse verwaltet. ${ }^{6}(54)$

Es ist klar, daß weder die Definition von Scheringer/Sprenger noch die von Rechtziegler als für die Stamokap-Diskussion repräsentativ hingestellt werden kann. Die Entwicklung der Stamokap-Theorie muß vielmehr im Zusammenhang mit den jeweiligen historischen Bedingungen gesehen werden. Margaret Wirth hat diesen Entwicklungsgang seit dem Zweiten Weltkrieg kurz zusammengefaßt: (55) Festhaltend an Lenins These vom Zeitalter des Niedergangs des Kapitalismus, wurde zunächst die Unterordnung des Staates unter die Monopole als letzter Versuch interpretiert, die kapitalistische Herrschaft aufrechtzuerhalten. Nachdem die für $1957 / 58$ vorhergesagte große Weltwirtschaftskrise ausgeblieben war, entstand die Theorie von der Labilität des Gesamtsystems als neuem Ausdruck der NiedergangsThese. Mit zunehmender staatlicher Aktivität (mittelfristige Finanzplanung, konzertierte Aktion, Haushaltsreform) wurde schließlich der staatsmonopolistische Kapitalismus definiert als das Gesamtsystem des heutigen Kapitalismus, als qualitativ neue Entwicklungsstufe der kapitalistischen Produktionsweise.

Ungeachtet aber der Tatsache, daß es verschiedene Varianten der StamokapTheorie gibt und daß die in der BRD vorherrschende, verglichen mit dem theoretischen Niveau in der DDR oder in Frankreich, nicht unbedingt die qualifizierteste Version ist, ist im Rahmen dieser Anmerkungen ausschlaggebend,

- daß in der Stamokap-Theorie, so wie sie uns in der Bauernpolitik der DKP entgegentritt, „,die politisch-ökonomische Methode der Marx'schen Kapitalanalyse ... tendenziell ... durch eine deskriptiv-politizistische (und häufig moralisierende) Denunziation der Machtinteressen und Bestrebungen der sogenannten, Monopole“ (56) ${ }^{\text {" }}$ ersetzt wird;

- daß mit der Stamokap-Theorie die antimonopolistische Bündniskonzeption theoretisch gerechtfertigt wird.

Die wichtigste Konsequenz der Stamokap-Theorie für den Klassenkampf besteht darin, daß ,in der jetzigen Etappe der Widerspruch zwischen allen Volksschichten und dem Häuflein der Monopolherren" (57) vorherrschend ist. Scheringer/Sprenger wiederholen diesen Kernsatz an relevanten Stellen: einmal bei der Einschätzung des Bündnisses der Arbeiterklasse mit der Bauernschaft (,... Hauptwiderspruch des spätkapitalistischen Systems, dem Widerspruch zwischen Monopolen und Volk ..."(58)), sodann bei der Behandlung der Großbauern (,Der

54) Marx/Engels: Manifest der Kommunistischen Partei, a. a. O., S. 464.

55) Margaret Wirth, Stamokap, Referat, gehalten am 9. 4. 1973 in Stuttgart. - Vgl. auch ihren Beitrag in diesem Heft.

56) Korte u. a., a. a. O., S. 6. - Vgl. in diesem Zusammenhang allgemein den Aufsatz von C. Möcklinghoff: Aspekte der Geschichte und Theorie der Bündnispolitik der KPD und DKP.In: Probleme des Klassenkampfs, Nr。 4 und 5 (Sept. u. Dez. 1972), S. 113-149 bzw. 1-44.

57) Erklärung der Beratung von Vertretern der kommunistischen und Arbeiterparteien, zitiert bei Scheringer/Sprenger, a. a. O., S. 151.

58) Scheringer/Sprenger, a. a. O., S. 153. 
entscheidende Widerspruch im Spätkapitalismus ist jedoch der Widerspruch zwischen Monopolen und Volk." $\left.{ }^{\text {" }}(59)\right)$.

Die Einordnung der Großbauern bereitet in der Tat die meisten Schwierigkeiten bei der Anpassung der Situation der BRD-Landwirtschaft an die Theorie des Stamokap. Unter Berufung auf das bereits angeführte Zitat von Lenin (vgl. Armerkung 20) schließen Scheringer/Sprenger aus der Abnahme der Landarbeiter, daß , in gewissem Sinne" ein ,Ubbergang von einem kapitalistischen Produktionsverhältnis zu Verhältnissen der einfachen Warenproduktion" (60) stattfindet, wăhrend sie, wie oben dargestellt, auf der anderen Seite die Degradierung der Mittel- und sogar Großbauern zu ,De-Facto-Lohnarbeitern " $(61)$ konstatieren. Indem die ganze Bauernschaft unter dem Begriff, ,Volk ${ }^{66}$ subsumiert wird, erweist es sich als schwierig, so es sich nicht erübrigt, Übergangsklassen, die im Prozeß der Durchdringung der Landwirtschaft auftauchen, $\mathrm{zu}$ analysieren. Bäuerliche „DeFacto-Lohnarbeiter" und bäuerliche einfache Warenproduzenten sind ja gleichermaßen „Volk“, das den Monopolen gegenübersteht.

Die „objektive“ Einbeziehung der Großbauern in das große Lager der antimonopolistischen Kräfte gelingt Scheringer/Sprenger mit einem einfachen formallogischen Kniff: „Die Großbauern werden immer mehr von den Monopolen beherrscht, so daß ihre Grundinteressen in einen immer tieferen Widerspruch zu den Monopolen geraten und sich demzufolge objektiv mit denen der Arbeiterklasse und der werktätigen Bauern treffen."(62) Mit anderen Worten: die Großbauern befinden sich im Widerspruch zu den Monopolen, die Arbeiterklasse und die werktätigen Bauern befinden sich im Widerspruch zu den Monopolen, ergo haben Großbauern einerseits und werktätige Bauern und Arbeiterklasse andererseits dieselben Interessen. In ähnlicher Weise gelingt es dem MSB Spartakus, die Agrarstudenten in das antimonopolistische Bündnis einzubeziehen: ausgehend von der Tatsache, daß Arbeiter und Bauern mit dem Märchen von der Lohn-Preis-Spirale gegeneinander ausgespielt werden, wird festgestellt, dab die Studenten ,wie alle anderen Bevölkerungsschichten von den Preistreibereien der Monopole betroffen sind“", um zu dem Schluß zu kommen: „Gerade hier zeigt sich deutlich: Arbeiter, Bauern und Studenten stehen demselben Gegner gegenüber." (63)

Auf der Ebene der Arbeiterpolitik ließe sich mit demselben Recht behaupten, daß sich die Interessen der kleinen Industrieunternehmer (analog den ,kleinen kapitalistischen Unternehmern in der Landwirtschaft, die einige Lohnarbeiter ausbeuten" (64)), die ja tatsächlich auch von den Monopolen bedroht werden, „objektiv mit denen der Arbeiterklasse ... treffen. ${ }^{66}$ Scheringer/Sprenger stellen diese Behauptung auch wirklich auf: „Tatsächlich gibt es objektiv keine Gemeinsamkeit der Interessen der Bauern, Handwerker, Einzelhändler sowie der kleinen

59) Ebenda, S. 169.

60) Ebenda, S. 78.

61) Ebenda, S. 95.

62) Ebenda, S. 79.

63) MSB Spartakus, Programm für das gemeinsame Handeln der Studenten, herausgegeben vom Bundesvorstand des MSB Spartakus, Bonn, März 1973, S. 58.

64) Scheringer/Sprenger, a. a. O., S. 158. 
und mittleren Unternehmer mit dem Großkapital. “(65) Gemeinsame antimonopolistische Interessen aller Volkskräfte werden also pauschal und kategorisch in den Stand der ,Objektivität ${ }^{\text {" }}$ erhoben, die Profitinteressen der ,kleinen“ Kapitalisten fallen unter den Tisch. Demgegenüber stellt Lambert fest: „Natürlich ist auch der kapitalistische Landwirt nicht von dieser Infiltration (der internationalen Firmen d. V.) geschützt. In den meisten Fällen findet er sich damit ab, indem er sich an diesen Gruppen beteiligt und zugunsten des einen oder anderen Stellung bezieht, wie Z. B. die Zuckerrübenbauern angesichts der Konzentration der Zuckerindustrie. Kurz gesagt, es handelt sich um normale Beziehungen zwischen Kapitalisten unterschiedlicher Stärke. " (66) (Hervorhebung d. V.)

Die These von der gesamten Bauernschaft als antimonopolistischer Kraft, der wir hier als Ausfluß der Stamokap-Theorie begegnen, ist nicht ganz neu. Im Zusammenhang mit der Bündnisfrage sagte Bucharin in der bereits erwähnten Rede: „Der frühere Grundsatz der Organisation hatte als Hauptaufgabe: Konstituierung der eigenen Klasse... Aber in einer Epoche, in der als unmittelbare Aufgabe die Eroberung der politischen Macht vor uns steht, stehen vor uns weitergehende Aufgaben. Da ist vor allem die Bündnisfrage."(67) Und über die Konflikte der gesamten Bauernschaft mit dem Kapital: „Die Kluft zwischen dem Bauerntum und der Bourgeoisie in den sogenannten zivilisierten Ländern erweitert sich in zwei Hauptrichtungen: einmal in der Richtung der Steuerpolitik ... und dann in der Richtung der ökonomischen Politik, der Syndikate und Trusts. Schließlich als drittes Moment kommt das der Kriegsgefahr hinzu." (68) Zum einen befinden wir uns gegenwärtig nicht unmittelbar vor der Machtergreifung durch das Proletariat, zum anderen betont Bucharin im Gegensatz zur DKP, die diese „Kluft zwischen dem Bauerntum und der Bourgeoisie" zum alleinigen- und Hauptwiderspruch macht: „Die Widersprüche zwischen den Großagrariern und den Bauern und die zwischen den Bauern und dem Großkapital sind verschiedenartiger Natur. Hinzu kommen Gegensätze, die innerhalb des Bauerntums selbst wirksam sind." (69) In den dem Referat Bucharins folgenden "Thesen zur Bauernfrage" wird die Großbauernschaft als „,Reserve der antiproletarischen Kräfte gegen das Proletariat"(70) bezeichnet, und selbst die Mittelbauern werden als nur bedingt bündnisfähig eingeschätzt, wenn die kommunistischen Parteien aufgefordert werden, ,gestützt auf . . die besitzlosen und wirtschaftsschwachen Schichten der Bauernschaft... schon jetzt eine energische Arbeit auch zur Neutralisierung der mittleren Bauernschaft (zu) führen." (71)

65) Ebenda, S. 153.

66) Lambert, a. a. O., S. 43.

67) Bucharin, a. a. O., S. 23.

68) Ebenda, S. 30.

69) Ebenda.

70) Ebenda, S. 40.

71) Ebenda, S. 58. In dieser Kede vor der Komintern heißst es in der 29 . These: „In solcher Weise müssen sie (der proletarische Staat und die herrschende proletarische Partei - d. V.) die Organisationen der landwirtschaftlichen Tagelöhner, der besitzlosen Landbevölkerung und der Mittelbauern zusammenschließen und auf jede Weise unterstützen, als 
In der Theorie vom Stamokap erhält der Staat eine neue Dimension: wird er durch die Verschmelzung mit den Monopolen quasi zum reellen Gesamtkapitalisten, so wird er natürlich auch zum unmittelbaren Klassengegner. Im Stamokap bedeutet der Kampf des Volkes gegen die Monopole also auch zugleich Kampf des Volkes gegen den Staat, und jeder ökonomische Kampf wird so a priori zum politischen Kampf. „Der Politik gehört das Primat, und die Machtfrage tritt bei allen Auseinandersetzungen zwischen Bauernschaft und Monopolbourgeoisie in den Vordergrund. Die ökonomischen Widersprüche in der Landwirtschaft, insbesondere der Widerspruch zwischen Bauernschaft und Monopolkapital, äußern sich immer mehr als politische Widersprüche zwischen der Agrarpolitik des Staates und den Interessen der Bauern" (72), schreibt Rechtziegler dazu.

Auch Scheringer/Sprenger stellen fest, daß sich die Hauptlosungen der Baism „eindeutig gegen die Agrarpolitik der EWG und der Bonner Regierung ${ }^{65}$ tich ten (73). Dennoch sind die Hauptforderungen der Bauern ökonomischer Natur, und dadurch, daß sie an den Staat gerichtet werden, werden sie nicht automatisch $z u$ politischen Forderungen. Darüberhinaus ist der Staat für die Bauern noch mehr als für andere Gesellschaftsgruppen politischer Ausdruck der Entfremdung des Menschen vom Menschen, einer Entfremdung, die im Falle der Bauern paternalistisch mystifiziert ist. Wenn sich der Bauer mit seinen Forderungen an den Staat wendet als an eine höhere Gewalt, die Gegensätze ausgleicht und für eine gerechte Verteilung des gesellschaftlichen Reichtums sorgt, so spricht aus dieser Haltung sicher nicht das Bewußtsein der ,politischen Gewalt " als "dem offiziellen Ausdruck des Klassengegensatzes innerhalb der bürgerlichen Gesellschaft. " (74.)

Der Umstand, daß die Bauern mit ihren Forderungen an den Staat appellieren, weil sie eben in keinem direkten Lohnverhältnis zum Kapital stehen und ihnen auf Grund ihrer isolierten Produktionsweise die Erfahrung der Ausbeutung durch das Einzelkapital fehlt, wird in einer solchen Interpretation als Beweis dafür genommen, daß die Bauern den Staat als direkte Instanz der Monopole begreifen und ansprechen. Zwar ist der Staat als ideelier Gesamtkapitalist tatsächlich gemeinsamer Gegner der Arbeiterklasse und der nicht-kapitalistischen Bauernschaft, aber bei der DKP wird die Gemeinsamkeit auf einer Ebene hergestellt, auf der sich der offensive Kampf der Arbeiterklasse gegen den „Ausschuß, der die gemeinschaftlichen Geschäfte der ganzen Bourgeoisieklasse verwaltet"(75), vermischt mit der typischen Defensivhaltung einer mittelständischen Gruppe, die in dem Maße, in dem das Großkapital die politische Macht okkupiert, ihre parlamentarisch-demokratischen Finflußmöglichkeiten als instre. ment ihrer wirtschaftlichen Existenzbehauptung schwinden sieht. Da die fortschreitende Kapitalakkumulation und -konzentration die Ursache der Ruinierung

Gegengewicht zu den sich herausbildenden neuen bürgerlich-kapitalistischen Schichten der Bauernschicft."

72) Rechtziegler, Westdeutsche Landwirtschaft ..., a. a. O., S. 109.

73) Scheringer/Sprenger, a. a. O., S. 166.

74) Vgl. Marx, Das Elend der Philosophie, in: Marx/Engels, Werke, Bd. 4, Berlin 1964, S. 182.

75) Marx/Engeis, Manifest der Kommunistischen Partei, a. a. O. 
bäuerlicher Existenzen ist, lassen sich bei einem organisierten Widerstand, wie er beispielsweise in der "Notgemeinschaft Deutscher Bauer" anzutreffen war, durchaus antimonopolistische Akzente entdecken, aber es handelt sich dabei um den Widerstand mittelständiger Schichten gegen ihr Abgleiten in eine proletarische Klassenlage und nicht um eine Parteinahme an der Seite der Arbeiterklasse gegen den gemeinsamen Klassengegner.

Solch unterschiedliche Widersprüche im Verhältnis von Arbeitern bzw. in Deklassierung befindlichen Bauern zum Staat zu einer gemeinsamen antimonopolistischen Motivation zu verquicken, wird möglich, weil die Stamokap-Theorie den bürgerlichen Staat als eine über den sozialen Gruppen stehende, neutrale Macht impliziert, die von der Arbeiterklasse und ihren Verbündeten umfunktioniert werden kann, und zwar in dem Sinne, daß sie ihn nur zur Umverteilung des Volkseinkommens zwingen muß (dabei erscheint die Umverteilung der Rüstungskosten bei der DKP als Allheilmittel, als Finanzquelle für sämtliche Sozialreformen). Anstelle des Kampfes um die Verfugungsgewalt des gesellschaftlichen Produktes tritt der Kampf um die staatliche Verteilung: der Gegensatz zwischen Lohnarbeit und Kapital soll durch staatliche Vermittlung ausgeglichen, statt abgeschafft werden; das revolutionäre Subjekt wird zum staatlichen Unterstützungsempfänger. Grundlage dieser Strategie ist die Annahme, das vermehrte Eingreifen des Staatsapparates, die ,staatsmonopolistische Regulierung", sei prinzipiell fortschrittlich, zur Zeit zwar noch mit reaktionären Inhalten gefuillt, aber tendenziell für die ,Demokratie“" ausnutzbar. Demgegenüber stellt Margaret Wirth fest: „Doch dieser Apparat wird nicht den ,Interessen der Werktätigen' entfremdet; vielmehr ist der Staatsapparat im Kapitalismus notwendigerweise etwas Fremdes, weil er die Bedingungen der Profitproduktion und nicht die Entwicklung der Gesellschaft organisiert. ${ }^{66}$ (76) Die Auffassung der DKP vom bürgerlichen Staat und seinen Regulierungsmaßnahmen eröffnet den ,friedlichen Weg zum Sozialismus" als Eroberung der bestehenden Flanungsinstitutionen. Darin aber zeigt sich eine Verwandtschaft der Theorie des staatsmonopolistischen Kapitalismus mit Hilferdings Theorie vom ,organisierten Kapitalismus": beide gehen davon aus, daß das Anwachsen der Rolle des Staates in der Wirtschaft eine demokratische Regulierung des Wirtschaftsprozesses im Interesse der ganzen Bevölkerung ermöglicht, und das dies erreicht wird, indem man die Führungseliten auswechselt, an die Stelle der Kapitalfunktionäre Arbeiterführer setzt. So käme es im Hilferdingschen "Generalkartell ${ }^{\text {" }}$ zu einer systemimmanenten Selbstaufhebung des Kapitalismus. Demgegenüber verstand Lenin unter Monopol ein ,ökonomisches und außerökonomisches Machtverhältnis ..., das die ökonomischen Verwertungszwänge durch Machteinfluisse und durch die Instrumentalisierung des Staatsapparates für die Durchsetzung seines Profitinteresses überlagerte, keineswegs aber diese Verwertungszwänge selbst aurzuheben imstande war. ${ }^{66}(77)$

Verbunden mit der Rolle, die dem Staat in der Theorie des Stamokap zukommt, sind zwei weitere Aspekte der DKP-Agrarpolitik.

76) Margaret Wirth, Kapitalismustheorie ..., a. a. O., S. $126 \mathrm{f}$.

77) Korte i. a., a. a. O., S.6. 
Zum einen die Systemkonkurrenz. Der staatsmonopolistische Kapitalismus ist der DKP-Theorie zufolge einerseits das Ergebnis des veränderten Kräfteverhältnisses in der Welt, Reaktion auf das Erstarken des sozialistischen Lagers. Andererseits überdeckt durch seine Existenz der Systemkonflikt Imperialismus/realer-Sozialismus immer mehr die nationalen Klassenkonflikte. Das gilt auch für die staatsmonopolistische Agrarpolitik. Rechtziegler gebraucht die Begriffe „Klassenauseinandersetzung zwischen Sozialismus und Kapitalismus“ und „Systemauseinandersetzung zwischen Sozialismus und Kapitalismus" synonym (in letzter Konsequenz wird also der Klassenwiderspruch durch den Systemwiderspruch ersetzt, auch wenn dazu das Kunststück notwendig ist, aus Klassengesellschaften Klassen, aus heterogenen Sozialgebilden homogene zu machen) und schreibt über die Ursachen der „,wachstumsorientierten Agrarstrukturpolitik ${ }^{66}$, „Für den westdeutschen Imperialismus ist insbesondere eine Neuorientierung seiner Agrarpolitik notwendig geworden, weil die sozialistische DDR demonstriert, wie die ökonomischen und sozialen Probleme der technischen Revolution in der Landwirtschaft erfolgreich gemeistert werden können." (78) Auf derselben Seite noch nennt Rechtziegler allerdings einen plausibleren Grund: die Instrumente der Bonner ,wachstumsorientierten Agrarpolitik" werden eingesetzt, um den Widerspruch zwischen dem Wachstum der Produktivkräfte in der Landwirtschaft und der sie beengenden Agrarstruktur der BRD abzuschwächen.

Zum anderen bedeutet zwar die konsequent zu Ende gedachte Theorie des Stamokap, daß der Staat der unmittelbare, wirkliche Klassenfeind wird, andererseits hat er sich aber auch von den Gesetzen des Kapitals verselbstständigt und wird ,für die antimonopolistischen Kräfte funktionalisierbar" (79) (Vgl. oben S. 176). Die Errichtung der ,antimonopolistischen Demokratie" bedeutet dann nichts anderes, als daß der Staat von den "demokratischen Kräften" als Instrument gegen die Monopole eingesetzt wird. Die praktische Konsequenz solcher Vorstellungen kommt beispielsweise im Abschnitt „Finanzierung“ des DKP-Bauernprogrammes zum Ausdruck: „Die Deutsche Kommunistische Partei sagt den Bauern und Verbrauchern: Geld ist im Staatshaushalt genug vorhanden, um dieses demokratische Bauernprogramm Punkt für Punkt zu verwirklichen. Das Geld wird jedoch nicht im Interesse des Volkes verwendet. Den Hauptteil der Etatmittel verschlingen die Rüstungswirtschaft und die Profitsucht der Konzerne. Die Gewinne der Konzerne sind für die Modernisierung der Landwirtschaft heranzuziehen. “ (80) Und Rechtziegler empfiehlt den westdeutschen Bauern, „die Macht der Monopole zurückzudrängen und die staatliche Förderung demokratischer Produktionsgemeinschaften durchzusetzen. ${ }^{6}$ (81) An anderer Stelle macht er den Einsatz der Genossenschaften im antiomonopolistischen Kampf der Bauern von staatlicher Unterstützung abhängig: „Um dies zu verwirklichen, muß ihre (der Genossenschaften - d. V.) großzügige Unterstützung durch staatliche Mittel erkämpft

78) Rechtziegler, Westdeutsche Landwirtschaft ..., a. a. O., S. 35, 36.

79) SHB/SF, Zur Kritik der, gewerkschaftlichen Orientierung“ in der Studentenbewegung. In: ,links", Nr。33, Mai 1972.

80) Demokratisches Bauernprogramm der DKP, a. a. O, S. 41.

81) Rechtziegler, Westdeutsche Landwirtschaft ..., a. a. O., S. 54. 
werden. Nur so können die Genossenschaften ein echtes Gegengewicht gegen die Macht der hochkonzentrierten Industrie und des Handels erlangen. ${ }^{6}{ }^{(82)}$

An diesem Punkt zeigt sich, daß die Stamokap-Theorie gezwungenermaßen in einer Sackgasse landen muß: die Zweieinigkeit Staat/Monopole macht es notwendig, daß der reelle Gesamtkapitalist sich selbst vernichtet, indem er als Staats-Haupt seine Glieder, die Monopole, ,zurückdrängt und schließlich überwindet."

Neuorientierung der Bündnisfrage im Lichte der Theorie des staatsmonopolistischen Kapitalismus

Um diesen Aspekt der DKP-Bauernpolitik auf den Begriff zu bringen, würde es eigentlich genügen, Rechtzieglers Charakteristik der neuen Situation aufzugreifen: „Bündnisfähigkeit aller Schichten der bäuerlichen Bevölkerung.“(83) Im Grunde bedeutet das, daß die Bündnisfrage im Stamokap gar nicht mehr existiert, nur noch aus marxistisch-leninistischer Tradition erörtert wird. Ein Bündnis im klassischen Sinne setzt unterschiedliche Interessen von Bündnispartnern gegenüber dem Proletariat voraus, die DKP-Theoretiker wiederholen aber unaufhörlich, daß die Arbeiterklasse und die Bauernschaft objektiv dieselben Interessen haben.

Danach zählen zu den objektiven Gemeinsamkeiten von Bauern und Arbeitern die Tatsachen, daß beide zusammen die „Hauptträger der materiellen Produktion“ sind (84) und daß ,Arbeiter und Bauern für die Erhaltung des Friedens und der Demokratie“(85) sind. In ähnlicher Richtung argumentiert auch der DKP-Vorsitzende Kurt Bachmann im Editorial der ersten Ausgabe der Landrevue: „Ausbeutung, Gewalt, Gefährdung des Friedens - davon sind die Bauern ebenso betroffen wie die Arbeiter. Aus dieser gemeinsamen Bedrohung bestehen die Grundlagen des Bündnisses zwischen Arbeiterklasse und der Bauernschaft.“(86) Scheringer/Spręnger schreiben auf den Leser ein: „In Wirklichkeit beruht das Bündnis auf den objektiv gleichen Interessen der Arbeiter und Bauern“ (87), Arbeiter und Bauern haben ,,durch ihre soziale Lage als Werktätige gemeinsame Interessen“ (88) und „Arbeiter und Bauern gehören darum zusammen, weil sie gleiche Interessen verbinden“ (89). Die Beschwörung der ,objektiv gleichen Interessen" enthebt allerdings nicht der Frage, weshalb das Bündnis nicht längst zustande gekommen ist, „wie es kommt, daß die vom kapitalistischen System erdrückte Bauernschaft sich politisch konservativ, als Hüter der bestehenden Ordnung verhält.“.'(90)

82) Rechtziegler, Neue Entwicklungstendenzen ..., a. a. O., S. 179.

83) Rechtziegler, Westdeutsche Landwirtschaft ..., a. a. O., S. 98.

84) Demokratisches Bauernprogramm der DKP, a. a. O., S. 7.

85) Rechtziegler, Westdeutsche Landwirtschaft ..., a. a. O., S. 108.

86) DKP-Landrevue, Jahrgang 1, Nr. 1/71.

87) Scheringer/Sprenger, a. a. O., S. 148.

88) Scheringer/Sprenger, a. a. O., S. 150.

89) Ebenda, S. 153.

90) Lambert, a. a. O., S. 9. 
Freilich haben Bauern und Lohnarbeiter objektiv gesehen die gleichen Interessen für ihre ökonomische und politische Zukunft, da die gegenwärtige Lage der Lohnarbeiter dem Bauern nur das Bild seiner unvermeidlichen Zukunft entgegenhält. Anders aber als der Lohnarbeiter hat der Bauer Privateigentum, also etwas zu verlieren, und dementsprechend Eigentumsillusionen. Das jedoch spricht die DKP nicht aus, denn in ihrer antimonopolistischen Agitation muß sie traditionell klassenkämpferische Argumente vermeiden, damit das breite Bündnis mit dem bäuerlichen Kleineigentum zustande kommt, muß sie sich gar zur Schützerin des bäuerlichen Privateigentums machen.

Die objektiv gleichen Interessen von Arbeitern und Bauern setzen natürlich auch voraus, daß die gesamte Bauernschaft für sich schon objektiv einheitliche Interessen hat. Auf welcher Grundlage werden die verschiedenen bäuerlichen Klassen bei der DKP zu einer einheitlichen „Bauernschaft“" zusammengefaßt? (Vgl. oben Anm. 46)

Die Behauptung, das sei möglich, weil alle Bauern einen gemeinsamen Gegner haben: die Monopole, ist in sich anfechtbar, darüber hinaus aber sicher nicht ausreichend, um die Gemeinsamkeit aller Bauern objektiv zu rechtfertigen. Im Manifest der Kommunistischen Partei heißt es bezüglich der bäuerlichen Klassen im feudalen Produktionsverhältnis: „Gutsherren, Freibauern, Fronpflichtige, Leibeigene, Landarbeiter stehen den Städtern antagonistisch gegenüber; dennoch sind sie keine Klasse, sondern quer durch sie geht die Klassenspaltung." (91) Und im „Achtzehnten Brumaire“ schreibt Marx zur Bauernfrage in der frühkapitalistischen französischen Gesellschaft: „Die Parzellenbauern bilden eine ungeheure Masse, deren Glieder in gleicher Situation leben, aber ohne in mannigfache Beziehung zueinander zu treten... Insofern Millionen von Familien unter ökonomischen Existenzbedingungen leben, die ihre Lebensweise, ihre Interessen und ihre Bildung von denen der anderen Klassen trennen und ihnen feindlich gegenüberstehen, bilden sie eine Klasse. Insofern ein nur lokaler Zusammenhang unter den Parzellenbauern besteht, die Dieselbigkeit ihrer Interessen keine Gemeinsamkeit, keine nationale Verbindung und keine politische Organisation unter ihnen erzeugt, bilden sie keine Klasse." (92)

Kurz: die Kriterien für die Existenz einer „Klasse für sich“, nämlich Hinausgreifen ïber lokale Begrenztheit durch Verbindung, Koordination und Organisation und der bewußt gewordene Gegensatz der eigenen zu den Interessen der anderen Klassen, werden von der seitens der DKP zur Klasse ernannten „Bauernschaft" nicht erfüllt. Aber auch die Kriterien einer „Klasse an sich", von denen Marx als wichtigste die „Dieselbigkeit der Revenuen und Revenuequellen" (93) (also Arbeitslohn, Profit und Grundrente als Einnahmequeilen der Lohnarbeiter-, Kapitalisten- und Grundbesitzerklasse) nannte, werden von den Bau-

91) Marx/Engels, Manifest der Kommunistischen Partei, a. a. O.

92) Marx, Der Achtzehnte Brumaire des Louis Bonaparte, In: Marx/Engels, Werke, Bd. 8, Dietz Verlag, Berlin 1969, S. 198.

93) Marx, Das Kapital Bd. III, in: Marx/Engels, Werke, Bd. 25, Dietz Verlag, Berlin 1970, S. 893. 
ern in ihrer Gesamtheit nicht erfüllt, vielmehr kommen alle drei Revenuearten in der Bauernschaft vor.

Grundlage der DKP-Bündnispolitik ist, daß alle Teile der Bauernschaft und die Arbeiterklasse "gemeinsam vom Monopolkapital ausgebeutet werden." (94) Die Argumentation geht etwa so: ,Die Kommunisten wissen, daß die übergroße Zahl aller Bauern in diesem Lande hart arbeitet und vom Monopolkapital ausgebeutet wird. Daß also die Bauernschaft nicht an der Seite des Monopolkapitals, sondern an der Seite der Arbeiterklasse ihre Interessen nach gesicherter Perspektive verwirklichen kann. ${ }^{\text {" }}$ (95)

Dagegen ist einzuwenden, daß einerseits die verschiedenen Übergangsklassen innerhalb der Bauernschaft und andererseits die Arbeiterklasse in durchaus unterschiedlichen Beziehungen zum Monopolkapital stehen. Der Begriff „Ausbeutung “ ist für die genau definierte Mehrwertaneignung durch den Kapitalisten, die Mehrwertenteignung des Arbeiters ist, reserviert. Diese Definition trifft nur für den aller Produktionsmittel ledigen Landarbeiter zu. Weitgehend gilt sie auch für den Arbeiter-Bauern (oder Nebenerwerbsbauer, wie die bürgerlichen Agrarpolitiker sagen), der zwar noch Produktionsmittel besitzt, sich seinen Hauptlebensunterhalt aber durch Verkauf seiner Arbeitskraft verdient; bedingt gilt sie auch für jene durch die Vertragslandwirtschaft proletarisierten Bauern, die neben ihrer Arbeitskraft nur noch wenige eigene Produktionsmittel einsetzen. (Vgl. oben S. 168) Bei allen anderen Beziehungen zwischen Bauern und Monopolen (und anderen aus der Landwirtschaft Nutzen ziehenden Schichten) handelt es sich um Ausplünderung, d. h. um Mehrwerttransfers in der Zirkulationssphäre. Dazu zählen die Beträge, die über die Preisschere (96) von der Landwirtschaft zu den Monopolen fließen, die Beträge, welche die Nahrungsmittelindustrie und der Agrarhandel zwischen Bauern

94) Demokratisches Bauernprogramm der DKP, a. a. O.

95) Hermann Sittner, Bündnis - weil die Interessen gleich sind. DKP-Landrevue, Jahrgang 1, Nr. 4/71.

96) Die Preisschere ist ein alter Begriff aus der marxistischen Diskussion der Agrarfrage. Bucharin sagte z. B. in seiner Rede ,Über die Bauernfrage" (a. a. O., S. 32): ,Eine der wichtigsten Ursachen der Schere ist die Politik der monopolistischen Organe des Kapitalismus." Rechtziegler beschreibt das Wirken der Preisschere in der BRD-Landwirtschaft so: "Geht man davon aus, daß die westdeutschen Bauern an der allgemeinen Preisentwicklung hätten teilnehmen können, die Agrarpreise also im gleichen Tempo gewachsen wären wie die landwirtschaftlichen Betriebsmittelpreise, dann hätte die Summe der Verkaufserlöse der Landwirtschaft zwischen 1950/51 und 1967/68 nicht 341,8 Milliarden DM, sondern 394,2 Milliarden DM betragen. Die Differenz, also -52,3 Milliarden DM, wurde den westdeutschen Bauern seit der Gründung der Bundesrepublik entzogen und zugunsten des Monopolkapitals umverteilt." (Rechtziegler, Westdeutsche Landwirtschaft ..., S. 29).

Poppinga schreibt dazu (Zur Agrarfrage in der Bundesrepublik, in: Lambert, a. a. O., S. 130): „Die bisherigen Theorien der Agrarpreisschere, also die Gegenüberstellung von Preisen für Agrarprodukte und Produktionshilfsmittel, sind aber kaum brauchbar, weil die stets stattfindenden Veränderungen der Arbeitsproduktivität und der Zahl der Arbeitskräfte nicht berücksichtigt werden."

Die Preisscheren-Theorie hat außer diesen statistischen noch andere Mängel: sie kann nur das globale Verhältnis zwischen der gesamten Landwirtschaft und der ihr vorgelagerten Betriebsmittelindustrie beschцeiben. Sie muß unberücksichtigt lassen, daß der Großbe- 
und Verbrauchern abschöpfen und die Leistungen an das Bankkapital. (Die Grundrente als eine verwandelte Form des in 'der Landwirtschaft erzeugten Mehrwerts, die vom Pächterkapitalisten an den Grundeigentümer gezahlt wird, ist für die BRD atypisch. Typischer dagegen ist die Grundreww - Pacht -, die der selbstständige Warenproduzent oder, ,integrierte "Bauer an einen Grundeigentümer - oft ein ehemaliger Bauer, aber auch Kirchen usw. - zahlt. Bei dieser Form der Landwirtschaft umfaßt die Grundrente einen Teil der eigenen Mehrarbeit des Bauern und unter Umständen sogar Teile seines ,Arbeiterlohnes ${ }^{66}, \mathrm{~d} . \mathrm{h}$. des Teils, der zur Reproduktion seines Lebens und seiner Familie notwendig ist (97). Ausplünderung ist auch dies.)

Scheringer/Sprenger fassen diese Formen der Ausplünderung pauschal unter der Überschrift ,Wie werden die Bauern ausgebeutet?"(98) zusammen, während Rechtziegler im Detail am Ausdruck Ausplünderung festhält (er spricht $z$. B. von der „Ausplünderung der Landwirtschaft durch die Monopolbourgeoisie über die Preisschere" (99), dann aber bei den Gemeinsamkeiten von Arbeiterklasse und Bauernschaft ebenfalls schreibt: „Arbeiter und Bauern werden durch das Monopolkapital ausgebeutet.“ $(100)$

trieb beispielsweise durch Mengenrabatte auch auf diesem Gebiet Vorteile (besser gesagt: weniger Nachteile) gegenüber dem Kleinbetrieb hat, und sie kann auch den Einfluß der Genossenschaften als Großbezieher von Produktionsmitteln nicht quantifizieren. Für die am weitesten eritwickelten Formen der Vertragslandwirtschaft, bei denen ein und derselbe Konzern die Produktionsmittel (etwa Futtermittel) liefert und den den Marktpreis ersetzenden ,Verrechnungspreis“ der Produkte bestimmt, ist sie nicht mehr brauchbar. Die Preisscheren-Theorie kann auch nur einen Teil der komplizierten ökonomischen Beziehungen zwischen der Gesamtlandwirtschaft und der übriger Volkswirtschaft erklären, sie reicht nicht aus, die Frage eindeutig zu beantworten, ob und in welchem Ausmaß Industrie und Handel auf Kosten der Landwirtschaft akkumulieren. Sicher ist nur, daß sich durch die Preisschere der Anteil der Bauern am in der Landwirtschaft erzeugten Mehrwert zugunsten von in der Zirkulationssphäre realisierten Profiten der Betriebsmittelkonzerne schmälert.

Im Zusammenhang mit der Stamokap-Theorie ist folgender Umstand interessant: die Preisschere wird durch die staatliche Subventionspolitik teilweise ausgeglichen, deren Aufgabe es ist, ,die Schranke, die das Monopolkapital der Akkumulation in der Landwirtschaft durch die Ausplïnderung setzt, zu überwinden und deren Investitionstätigkeit voranzutreiben。" (Rechtziegler, Westdeutsche Landwirtschaft ..., a. a. O., S. 31). Für das verschmolzene Staat/Monopol-Gebilde der Stamokap-Theorie wäre es sicher einfacher, die Landwirtschaft weniger auszuplündern und ihr so eine subventionsfreie erweiterte Reproduktion zu erlauben. Aber die Verhältnisse sind komplizierter: es besteht ein Widerspruch zwischen den kurzfristigen Profitinteressen der Einzelmonopole und dem langfristigen Interesse des Gesamtkapitals an einer akkumulationsfähigen Landwirtschaft. Der Staat entschärft mit seiner Subventionspolitik diesen Widerspruch. Gleichzeitig erlaubt die Ausplünderung der Bauern, indem sie diese zwingt, mit Fremdkapital die erweiterte Reproduktion aufrechtzuerhalten, einer weiteren Kapitalfraktion, dem Finanzkapital, sich über die Kreditvergabe an der Landwirtschaft zu bereichern.

97) Vgl. Marx, Das Kapital, Bd. III, a. a. O., S. 638.

98) Scheringer/Sprenger, a. a. O., S. 98.

99) E. Rech tziegler, Westdeutsche Landwirtschaft ..., a. a. O., S. 29.

100) Ebenda, S. 108. 
Der Konzentration auf die „Ausbeutung“ von Bauern und Kleineigentum durch die Monopole und der Elimination des marxistischen Ausbeutungsbegriffes aus der Agitation liegt dieselbe Überlegung zugrunde, wie der noch näher zu erläuternden opportuinistischen Haltung der DKP-Bauernpolitik in der Eigentumsfrage: „Soll das Kleineigentum nicht wieder verunsichert werden, muß der Gegensatz von Lohnarbeit und Kapital... aus der Agitation der Partei so weitgehend wie möglich eliminiert werden. Denn die tradtionelle Gestalt des Klassenkampfes birgt die Gefahr, daß das ,breite Bündnis' mit dem Kleineigentum wieder gesprengt und die Klasseneinheit der Bourgeoisie wieder hergestellt würde“ (101). Margaret Wirth stellt bei ihrer Kritik des von dem DDR-ökonomen Kurt Zieschang eingefuihrten Begriffes, ,gesellschaftliche Ausbeutung" fest, daß die politische Bedeutung dieser These darin liegt, ,daß so dem Arbeiter (und auch dem Bauern - d. V.) in Übereinstimmung mit der Volksfrontstrategie eine partielle Interessenidentität mit der nichtmonopolistischen Bourgeoisie suggeriert werden kann" (102).

Wird in einer programmatischen, der Propaganda dienenden Schrift wie dem DKP-Bauernprogramm die Bündnisfähigkeit der gesamten Bauernschaft schlicht unterstellt und das „Bündnis der Arbeiterklasse mit der Bauernschaft" proklamiert, so wird die Frage bei Scheringer/Sprenger und auch bei Rechtziegler doch etwas differenzierter behandelt. Scheringer/Sprenger konstatieren, daß bei den Bauern die „Vorstellungen über ihre gesellschaftliche Rolle oft die alten geblieben sind“ (103), und räumen sogar den Halbprọletariern (Arbeiter-Bauern) eine besondere Stellung innerhalb der Arbeiterklasse ein, nämlich als einer „Gruppe mit spezifischen ideologischen und politischen Wesenszügen“, bei der ,vielfach noch die Ideologie des Kleineigentümers" vorherrscht (104). Rechtziegler macht das Zustandekommen des Bündnisses vom ,subjektiven Faktor, also vom Klassenbewußtsein sowohl der Arbeiter als auch der Bauern abhängig" (105). Indem die Unterschiede zwischen Arbeitern und Bauern „Vorstellungen“ und „Ideologien“, also dem ,subjektiven Faktor" zugeschrieben werden, fällt es leicht, sie im Lichte der "objektiven Gemeinsamkeiten" zu vernachlässigen; zugleich wird so eine oberflächliche Begründung dieser „Vorstellungen“ gegeben (aus Manipulation statt aus realen Interessen).

So wehrt sich Hamza Alavi in seiner Auseinandersetzung mit der indischen Bauembewegung zu Recht gegen die Behauptung, die geringe Militanz hier der armen Bauern. sei lediglich auf ihre rückständige Mentalität, auf ihre veralteten „Vorstellungen über ihre gesellschaftliche Rolle“ zurückzuführen. Er schreibt: „,Die Treue der armen Bauern und Landarbeiter zu ihren Herren ist nicht einiach das Resultat subjektiver Faktoren wie etwa der ,rückständigen Mentalität usw. Sie basiert vielmehr auf der objektiven Tatsache, daß sie um eines gesicherten Lebensunterhaltes willen von ihren Herren abhängen." Und: „Die subjektive

101) Korte u. a., a. a. O., S. 6.

102) Margaret Wirth, Kapitalismustheorie .... a. a. O., S. 69.

103) Scheringer/Sprenger, a. a. O., S. 161.

104) Ebenda, S. 156.

105) E. Rechtziegler, Westdeutsche Landwirtschaft ..., a. a. O., S. 100. 
Rückständigkeit des Bauern ist in objektiven Faktoren verwurzelt." (106) Alavi sieht in der Abhängigkeit von seinem Herrn auch einen „fundamentalen Unterschied zwischen der Situation des armen Bauern und derjenigen des Industriearbeiters": während der Industriearbeiter bei der Wahl seines Arbeitsplatzes in gewissem Umfang beweglich ist, bleibt der Bauer auf Grund seiner ökonomischen Abhängigkeit an seinen Feudalherren, aber auch, soweit er welches hat, an sein Bodeneigentum gefesselt. Dasselbe stellte Lenin 1913 für die bereits voll im Prozess kapitalistischer Integration befindliche deutsche und österreichische Landwirtschaft fest: „Der Bauer ist durch das komplizierte Netz der kapitalistischen Abhängigkeit mehr gebunden, mehr gefesselt als der Lohnarbeiter.“(107) Ähnliches läßt sich über die „Ideologie der Kleineigentümer" der bundesrepublikanischen Bauern sagen. Für die verschiedenen Teile der Bauernschaft trifft in unterschiedlichem Ausmaße $\mathrm{zu}$, daß ihr Festkleben am Grund und Boden, ihr Mißtrauen gegenüber der Stadt und insbesondere der Arbeiterklasse nicht bloße Ideologie ist, sondern in ihrem objektiven Interesse an ökonomischer Sicherheit wurzelt. Abgesehen von günstigen Baulandgeschäften behält auch der Bauer, der seinen Betrieb ganz aufgibt, seinen Boden; in der Regel verkauft er ihn nicht, sondern verpachtet ihn. Die Bauern, besonders die älteren, haben diese ökonomische Sicherheit in den Kriegen und Wirtschaftskrisen schätzen gelernt und sie beobachten auch heute, wie es dem aller Produktionsmittel beraubten Arbeiter geht, wenn er auf Kurzarbeit gesetzt oder entlassen wird. Ahnlich wie der gegenwärtige Zustand des Arbeiterbewußtseins, der keineswegs mit der von der Stamokap-Theorie konstatierten ständigen Verschärfung der Widersprüche einhergeht, mit der weiten Verbreitung des Opportunismus erklärt wird, wird in der Bauernpolitik z. B. „die Verleumdung der Landwirtschaft in den sozialistischen Ländern"(108) für das Mißtrauen der Bauern gegenüber der Arbeiterklasse und der DKP verantwortlich gemacht. Die Entstehung von Klassenbewußtsein ist damit nicht mehr abhängig von den ökonomischen Gesetzen und ihrer Entwicklung, sondern von der Manipulationsfähigkeit des Kapitals.

Die Bündnispolitik der DKP vernachlässigt die Zwiespaltigkeit in der objektiven Situation des Bauern, die darin besteht, daß er zugleich Werktätiger und Besitzer von Produktionsmitteln ist (109); der Arbeiter-Bauer und der Vertragsbauer in der höchsten Stufe der vertikalen kapitalistischen Integration sind zugleich Lohnabhängige und Produktionsmittelbesitzer. Deshalb ist für den Vertragsbauern auch der Begriff „Heimarbeiter“" angebrachter als „De-Facto-Lohnarbeiter“. Der Vertragsbauer wird im besten Fall zum Juniorpartner des Großkapitalisten, seine Landwirtschaft zum Zulieferbetrieb, im schlechtesten Fall zum Heimarbeiter mit einer handfesten materiellen Sicherheit in der Reserve. Die Merkmale des landwirtschaftlichen Heimarbeiters, die ihn auch im Stadium größter Abhängigkeit von den Konzernen vom Lohnarbeiter unterscheiden, sind diese materielle Sicherheit, das Gefesseltsein an den Boden und die Tatsache, daß er zum Vorteil der

106) H. Alavi, a. a. O., S. 54/55.

107) Lenin, 1913, Der Kleinbetrieb in der Landwirtschaft, in: Lenin, Werke, Bd. 19, Dietz Verlag, Berlin 1971, S. 272.

108) MSB Spartakus, a. a. O., S. 58.

109) Rechtziegler geht darauf ein: Westdt. Landwirtsch., a. a. O., S. 99. 
Monopole mehr oder weniger die Investitionen bezahlt und das volle Produktionsrisiko trägt. Lenin sah in der Unterscheidung zwischen dem Bauern als Werktätigen und dem Bauern als Eigentümer und Händler das Wesen der sozialistischen Auffassung von der Agrarfrage (110). In den von Bucharin entwickelten Thesen zur Bauernfrage werden sowohl für die ,mittlere Bauernschaft ${ }^{66}$ als auch die ,Klein-

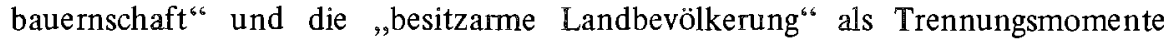
gegenüber der Arbeiterklasse die ,Interessen der privaten Warenwirtschaft " und das „Privateigentum" genannt. Die Bündnisfähigkeit dieser drei Gruppen wird daher folgendermaßen beurteilt: die Mittelbauern können neutralisiert werden, und ,wo der kapitalistische Druck besonders stark ist, oder wo er sich mit dem feudalen Druck verbindet... mit dem Proletariat zusammengehen. ${ }^{66}$ Die Kleinbauernschaft kann, da „,der Kleinwirt sich nicht selten als Lohnarbeiter verdingt,... für das Proletariat gewonnen und dessen entscheidender Verbündeter werden." Die besitzarme Landbevölkerung, bei der , das Trennungsmoment gänzlich unbedeutend ist ${ }^{66}$, stellt die Reserve des Proletariats dar. Die Landarbeiter schließlich sind zwar ein Teil des Proletariats, haben aber die Besonderheit der ,Zerstreutheit... im Arbeitsprozess" und des ,stark ,patriarchalischen" Charakters der ländlichen Verhältnisse." (111)

Bedeutet die Kritik an der Bündnispolitik der DKP, an der pauschalen Unterordnung der verschiedenen Widersprüche zwischen den Teilen der Bauernschaft und der Arbeiterklasse unter den Widerspruch Volk/Monopole, daß die Bauern alleine und nicht gegen die Monopole kämpfen sollen? Keineswegs. Eine erfolgreiche politische Arbeit unter den Bauern muß jedoch so differenziert ansetzen wie die Verhältnisse auf dem Lande komplex und mannigfaltig sind. So gebietet die zunehmende Abhängigkeit und soziale Degradierung der Mittelbauern, daß die daraus eventuell resultierende Militanz auch zur Mobilisierung der bereits resignierten Kleinbauern genutzt wird. Hamza Alavi hat am Beispiel der chinesischen Revolution nachgewieseh, daß sich die Mittelbauern zunächst am militantesten verhielten, weil sie unter der Terrorherrschaft der Kriegsherren und Steuereintreiber am meisten zu verlieren hatten (112). Ein ähnlicher Effekt könnte bei uns eintreten, wenn die Mittelbauern immer schneller und brutaler aus ihrer relativ selbstständigen Position auf den Weg der Proletarisierung gedrängt werden. Man darf die Analogie allerdings nicht zu weit treiben: während die Bauernschaft in der kapitalistischen Gesellschaft keine einheitliche Klasse ist, ist sie es - jedenfalls in gewisser. Weise - im Kampf gegen die feudalen Großgrundbesitzer (mit Ausnahme jener Schichten, etwa der russischen Kulaken, die außerhalb des Herrschaftsbereiches der Feudalherren stehen). Außerdem ist der Kampf gegen den Feudalismus offensiv, es geht um die Befreiung von der Leibeigenschaft, um den Aufstieg vom hörigen Pächter zum selbstständigen Bodeneigentümer. Der Kampf gegen das Vordringen des Kapitals in der Landwirtschaft ist dagegen zunächst

110) Siehe dazu: Lenin, Ökonomie und Politik in der Epoche der Diktatur des Proletariats. Werke, Bd. 30, S. 97.

111) Alle Zitate bei: Bucharin, a. a. O., S:-40/41.

112) Alavi, a. a. O., S. $28 \mathrm{f}$. 
defensiv. Die kleinen Warenproduzenten werden ihn, auf sich gestellt, ständisch führen, das heißt: Verteidigung ihrer Privilegien gegen das Absinken ins Proletariat. Hier liegt das wirkliche Arbeitsfeld der Bündnispolitik. Die Bauern müssen folgendes sehen: ,Da die kapitalistischen Produktionsverhältnisse auf unsere Proletarisierung hinauslaufen, müssen wir unseren Kampf gegen dieses System auch als Proletarier führen." (113)

Die Frage, ob der Kampf der Bauern ständisch vom Standpunkt des deklassierten kleinen Warenproduzenten oder proletarisch geführt wird, wirft auch das Problem der Führung durch die Arbeiterklasse auf. Die führende Rolle des Proletariats gegenüber den Bauern rechtfertigt sich aus der Tatsache, daß das Proletariat als einzige Klasse keine Bindung an das Privateigentum besitzt. Die werktätige Bauernschaft braucht die Führung des Proletariats im revolutionären Kampf also vor allem deshalb, weil sie an das Eigentum gefesselt ist. Das organisierte Proletariat kann auf Grund seiner Klassenlage den Kampf gegen die Diktatur der Bourgeoisie am konsequentesten führen, und „nur der Fall des Kapitals kann den Bauern steigen machen, nur eine antikapitalistische, eine proletarische Regierung kann sein ökonomisches Elend, seine gesellschaftliche Degradation brechen." (114) Dasselbe Zitat findet sich bei Rechtziegler, und er fügt hinzu: „Auch weil ihre Haltung durch diese Zwiespältigkeit (zugleich Werktätige und Besitzer von Produktionsmitteln - d. V.) bestimmt wird, kann ihre Befreiung nur im Bündnis mit der Arbeiterklasse und unter deren Führung erfolgen. " (115) Scheringer/Sprenger sprechen ebenfalls von der "führenden Rolle" der Arbeiterklasse (116). (An anderer Stelle begnügen sie sich mit der verschämten Formulie rung: ,Die Hauptverantwortung für dieses Bündnis trägt die Arbeiterklasse und ihre marxistische Partei." (117)) Dagegen klammert das Demokratische Bauernprogramm diese Frage ganz aus. Es gibt zwei Ursachen dafür, daß die DKP-Bauernpolitik das Problem der Führung der Bauern durch die Arbeiterklasse nicht oder nur nebenbei erwähnt:

- Im Volksfront-Eintopf der antimonopolistischen Kräfte treten die Klassen, ihre Bündnisse und daraus resultierende Fragen der Führung hinter dem „Volk" zurück. Die einheitliche Bauernschaft steht auf denselben objektiven, antimonopolistischen Grundlagen wie die Arbeiterklasse und bedarf deren Führung nicht mehr.

- Indem die DKP die Eigentumsfrage auf den Kopf stellt (wie im nächsten Abschnitt gezeigt werden soll), fällt das objektive Unterscheidungsmerkmal zwischen Bauern und Arbeitern, die Fesselung ans Privateigentum, und damit auch die Legitimation für die Führung der Arbeiterklasse weg.

113) Lambert, a. a. O., S. 68

114) Marx, Die Klassenkämpfe in Frankreich 1848 und 1850. Werke, Bd. 7, Berlin 1960, S. 84.

115) Rechtziegler, Westdeutsche Landwirtschaft ..., a. a. O., S. 99.

116) Scheringer/Sprenger, a. a. O., S. 151.

117) Ebenda, S. 162. 
In der Landrevue wird die „Stellung der DKP zum bäuerlichen Eigentum“ als "Fernstick des Demokratischen Bauernprogramms" bezeichnet (118).

In einer Zeit, in der die Frage insbesondere des Bodeneigentums im Brennpunkt der öfentlichen Diskussion steht, beeilt sich die DKP zu versichern: „Demokratische Landreform setzt die Erhaltung des bäuerlichen Eigentums voraus und sichert es. ${ }^{66}$ (119) Demokratische Landreform bedeutet, „das Land des feudalen und großkapitalistischen Grundbesitzes den werktätigen Bauern und Landarbeitern, ihren zwischenbetrieblichen und gemeinschaftlichen Einrichtungen zur Verfügung zu stellen。 ${ }^{6}$ (120) (Hervorhebung v. V.) Die Frage des Bodeneigentums wird also zunächst auf das Nebengeleise des in der BRD noch vorhandenen Großgrundbesitzes geschoben, wo man sich denn auch auf die historischen Beispiele der Landverteilung durch kommunistische Regierungen berufen kann (121).

Wie schwer sich die DKP mit der Eigentumsfrage tut, spiegelt sich schon in der Benennung ihres, ,agrarpolitischen Leitbildes" (wie die bürgerlichen Agrarpolitiker zu sagen pflegen): im Entwurf des Demokratischen Bauernprogrammes war von ,,modernen Bauernwirtschaften ${ }^{6}(122)$ die Rede, in der endgültigen Fassung heißt es dann „moderne bäuerliche Landwirtschaft" (123). Die Assoziation zur ,bäuerlichen Familienlandwirtschaft", jahrelanges Leitbild der Bonner Agrarpolitik und des Deutschen Bauernverbandes, stellt sich ein.

Die DKP motiviert ihren Einsatz für das bäuerliche Eigentum damit, daß ,dieses Eigentum... gegen die Enteignung durch den kapitalistischen Struktur-

118) DKP-Landrevue, Jahrgang 1 , Nr. $2 / 72$

119) Demokratisches Bavernprogramm der DKP, a. a. O., S. 17.

120) Ebenda, S. 18.

121) Nach dem 2. Weltirieg, als in den Westzonen die Agrarreform systematisch sabotiert wurde, war die Forderung nach Enteignung des Großgrundbesitzes sicherlich politisch relevanter als heute. Damals wurde sie sogar z. T. zur unumgänglichen Voraussetzung jeder Veränderung auf dem Lande gemacht (siehe z. B. H. W. Dölling in ,Wende der deutschen Agrarpolitik“" Deutscher Bauernverlag, Berlin 1950, S. 254: „,Die erste Maßnahme ist die entschädigungslose Enteignung des gesamten Großgrundbesitzes. Sie ist und bleibt eine der ersten Voraussetzungen zur Gesundung der westdeutschen Landwirtschaft." ) Unter Bedingungen wie denen des Junkertums in Ostelbien vor der DDR-Agrarreform ist die Frage der Aufteilung von enteignetem Großgrundbesitz politisch wichtig. Diese sogenannte, ausgleichende Bodennutzung" ist der Kern des 1917 auf dem 2. Gesamtrussischen Sowjetkongreß angenommenen „Dekretes über den Grund und Boden" (Lenin, Werke, Bd. 26, S. 249/50). Lenin sprach von ihr als von einer Ubergangsmaßnahme zum vollen Sozialismus und unter der Bedingung, daß die Diktatur des Proletariats errichtet ist, von eimem ,Schritt zum Sozialismus" (siehe: Lenin, Werke, Bd. 28, S. 315/16). Bucharin vertritt in einer Gegenüberstellung der russischen und der vingarischen Revolution dieselbe Ansicht, wenn er sagt: „Produktionstechnisch haben wir zwar verloren ... Aber das war der Preis, um den wir den Sieg erkauften, durch den wir .

deri Block zwischen Bauernschaft und Arbeiterklasse ... erlangten." (Bucharin, a. a. Q., 3.28).

DKR, Demokratisches Bauernprogramm, Entwurf, Düsseldorf o. J., S. 6.

Demokratisches Bauernprogramm der DKP, a. a. O., S. $10,18$. 
wandel zu verteidigen ist."(124) Scheringer/Sprenger betonen, daß es gälte, ,das Eigentum der Bauern auch als Einzelproduzenten gegen die Willkür der Monopole und gegen den kapitalistischen Strukturwandel (zu) verteidigen" (125) (Hervorhebung v. V.), obwohl sie an anderer Stelle Engels zitieren, der in „Die Bauernfrage in Frankreich und Deutschland“" schreibt: „Es ist die Pflicht unserer Partei, den Bauern immer und immer wieder die absolute Rettungslosigkeit ihrer Lage, solange der Kapitalismus herrscht, klarzumachen, die absolute Unmöglichkeit, ihnen ihp Parzelleneigentum als solches zu erhalten..."(126) (Hervorhebung v. V.). Die Formulierung mit dem Schutz des bäuerlichen Eigentums vor dem kapitalistischen Strukturwandel ist Opportunismus, denn die DKP weiß wohl auch, daß die Bauern, wenn sie von bäuerlichem Eigentum reden, genau dieses „Parzelleneigentum als solches" meinen und nicht das bäuerliche Eigentum der ,,modernen bäuerlichen Landwirtschaft", das nichts anderes sein kann als das De-Jure-Eigentum des Einzelbauern in der Genossenschaftslandwirtschaft mit de facto gemeinsamem Bodeneigentum. Dies wagt die DKP nicht auszusprechen. In der Frage des gemeinschaftlichen Bodeneigentums versteckt sich das Demokratische Bauernprogramm hinter einem ,usw.“: „Die demokratische Entwicklung der Zusammenarbeit ist auf die Dauer nur gewährleistet, wenn Schritt für Schritt gemeinschaftliches Eigentum an Maschinen, Gebäuden, Bewässerungs- und Trocknungsanlagen, Speichern, Silos usw. geschaffen ... . wird." (127) (Hervorhebung v. V.)

Man kann diesen Teil der DKP-Agrarpolitik nicht als Taktik entschuldigen. Wo die Taktik gegen das strategische Ziel, nämlich die Bauern für das Bündnis mi der Arbeiterklasse zu gewinnen, verstößt, wird sie gefährlich. Genau das ist der Fall, wenn mit der DKP-Eigentumspolitik die Bauern in ihrer Rolle als Eigentümer bestärkt werden. Marx und Engels haben den eingefleischten Eigentumssinn der Bauern als wesentliches Hindernis bei der Schaffung des Bündnisses mit der Arbeiterklasse hervorgehoben. Engels schreibt: „Je schwerer ihm der Kampf wird um sein gefährdetes Fetzchen Land, mit desto gewaltsamerer Verzweiflung klammert er sich daran fest ...“(128). Und Marx: „Der Eigentumstitel der Bauern ist der Talismann, womit das Kapital ihn bisher bannte, der Vorwand, unter dem es ihn gegen das industrielle Proletariat aufhetzt.“(129) Die Parole von der „Erhaltung des bäuerlichen Eigentums" hält darüberhinaus die jahrelang von der Bonner Agrarpolitik gepflegte Illusion aufrecht, alle Bauern könnten in ihrem Beruf und auf ihrem Betrieb bleiben. Rechtziegler geht auf diese Frage ein: „Soweit die

124) Ebenda, S. 18.

125) Scheringer/Sprenger, a. a. O., S. 103.

126) Zitiert bei Scheringer/Sprenger S. 63 - Scheringer/Sprenger befinden sich nicht ,in voller Übereinstimmung mit Friedrich Engels“. Engels schreibt zwar, wie sie zitieren (S. 103), daß sich die Arbeiterklasse dafür einsetzen muß, ,daß der Kampf der Kapitalisten und Großgrundbesitzer gegen die Kleinbauern schon heute mit möglichst wenig unrechtlichen Mitteln geführt und direkter Raub und Prellerei ... möglichst verhindert wird." Von Verteidigung des bäuerlichen Eigentums schreibt er nichts.

127) Demokratisches Bauernprogramm der DKP, a. a. O., S. 22.

128) Engets, Die Bauernfrage in Frankreich und Deutschland, in: Marx/Engels, Werke, Bd. 2. Dietz Verlag, Berlin 1963, S. 489.

129) Marx, Die Klassenkämpfe in Frankreich 1848 und 1850, a. a. O., S. 87. 
Freisetzung von Arbeitsk räften in der Landwirtschaft objektiv unumgänglich ist, muß sie im Interesse der Betroffenen ohne größere soziale Belastungen vollzogen werden. "(130) Das DKP-Bauernprogramm erwähnt an keiner Stelle (auch nicht in den Abschnitten über Schul- und Ausbildungswesen bzw. Sozialpolitik) das Problem der Umschulung und Schaffung industrieller Arbeitsplätze. Die DKP weiß, daß auch in der DDR, deren Verhältnisse sie sonst gerne zur Untermauerung ihrer Thesen heranzieht, eine Abwanderung aus der Landwirtschaft stattfindet: 1960 betrug die Zahl der Beschäftigten in der Land- und Forstwirtschaft der DDR 1,3 Millionen Personen 1970 waren es noch 997 100. Die jährliche Abwanderungsrate aus der Landwirtschaft liegt zwischen 2 und $3 \%$ (131).

Die DKP stellt die Eigentumsfrage in der Landwirtschaft auf den Kopf, indem sie den ständischen, nach rückwärts gerichteten Kampf der Bauern als kleine Warenproduzenten um die Erhaltung ihres Privateigentums a priori in einen antikapitalistischen, fortschrittlichen Kampf verkehrt: „Indem die Bauern um die Erhaltung ihres Eigentums kämpfen, kämpfen sie objektiv gegen die Monopole, ist ihr Kampf unter den gegebenen Umständen objektiv antimonopolistischer Kampf.“(132) Und in den „Thesen des Düsseldorfer Parteitages der Deutschen Kommunistischen Partei": „Seinem Charakter nach ist der Kampf der Bauern um die Verbesserung ihrer Einkommen, ihrer Arbeits- und Lebensbedingungen und um ihr Eigentum zugleich ein antimonopolistischer Kampf." (133)

Wie sieht es mit dem antimonopolistischen Kampf der Bauern um ihr Eigentum und um ihr Einkommen in Wirklichkeit aus? Zwei Beispiele - die Notgemeinschaft Deutscher Bauer und der Milchkrieg von Weiding - sollen beleuchten, wie gefährlich eine Einschätzung ist, die den Widerstand bäuerlicher Schichten gegen ihre Deklassierung von vorneherein zum antimonopolistischen Kampf erklärt.

Die Notgemeinschaft Deutscher Bauer (134) wurde im Februar/März 1963 gebildet und noch imselben Jahr aus dem Deutschen Bauernverband ausgeschlossen. Sie war in Niedersachsen und Schleswig-Holstein im Anschluß an große Bauerndemonstrationen entstanden und war anfangs ziemlich erfolgreich. Die Arbeitsgemeinschaft für westdeutsche und westeuropäische Landwirtschaft an der KarlMarx-Universität Leipzig analysierte die Notgemeinschaft und kam zu folgendem Schluß: „Die Notgemeinschaften sind die einzigen legalen Organisationen der Bauern, mit deren Hilfe sie ihre Interessen durchsetzen können. Sie bleiben nicht bei wirtschaftlichen und sozialen Forderungen stehen, weil wirkliche ökonomische Erfolge nur im Kampf gegen Imperialismus und Krieg errungen werden

130) Rechtziegler, Westdeutsche Landwirtschaft ..., a. a. O., S. 97, 98.

131) Hans Immler, Die Landwirtschaft in der DDR. Institut für angewandte Agrarpolitik an der Technischen Universität Berlin, 1971.

132) Wilhelm Sprenger, $\mathrm{Zu}$ den Ursachen der Bauerndemonstrationen. In: Marxistische Blätter, Frankfurt/M., Nr. 5, Spt./Okt. 1971, S. 74.

133) - Zitiert in DKP-Landrevue, Jahrgang 1, Nr. 4/71.

134) Die Ausführungen folgen Onno-Hans Poppinga, Zur Bauernfrage in Westdeutschland. In: Beiträge $7 u r$ Agrarfrage in der BRD. Plakat-Bauernverlag, Stuttgart 1972, S. 14, 15. 
können." (135)

Bei der Bundestagswahl 1965 rief die Notgemeinschaft ihre Anhänger auf, die NPD zu wählen; und in ihrem ,fortschrittlichen Programm" (136) wird die Forderung aufgestellt nach „Förderung der geistigen und sittlichen Kräfte des Bauerntums als Mutterschicht nationaler Kultur." Der „bekannte Bauernführer Petersen" (137) schließlich war Mitglied von NSDAP und SS, Referent und Landwirtschaftsrat im faschistischen Reichsnährstand und wurde 1967 als Agrarexperte der NPD in den Landtag von Schleswig-Holstein gewählt. Zweifellos tauchten bei der Notgemeinschaft auch antikapitalistische Vorstellungen auf, aber ihrer Entstehung nach - hauptsächlich in großbäuerlichen oder bis vor wenigen Jahren großbäuerlichen Regionen - war sie eine Bewegung deklassierter Bauern, mit der entsprechenden, auf die Rückkehr zu den alten Zuständen gerichteten Ideologie.

Der Milchkrieg in Weiding brach aus, als die Vertragsbauern des Milchwerkes Weiding der Allgäuer Alpenmilch AG, die zum Nestlé-Konzern gehört, sich zu einer „Aktion gerechter Milchpreis" zusammenschlossen. Bei Vertragsabschluß hatte das Milchwerk Weiding einen höheren Milchauszahlungspreis als die anderen Molkereien der Nachbarschaft; als die „Aktion“" Anfang 1971 begann, war es umgekehrt. Jetzt forderten die Bauern 46 Pfennig je $\mathrm{kg}$ Milch bei 3,7\% Fettgehalt. Es wurden Vollmachten gesammelt, die die „Aktion“ zum Sprecher der Bauern gegenüber der Alpenmilch legitimierten. Von 7000 Milchanlieferern des Werkes Weiding gaben ca. 5000 ihre Vollmacht, in einem Solidaritätsfonds kamen $70000 \mathrm{DM}$ zusammen. $\mathrm{Ab}$ Sommer 1971 berichtete die DKP-Landrevue ausfiuhrlich über die "Milli-Gaudi“ wie die beteiligten Bauern es nannten. Richard Scheringer signalisierte die Einschätzung der DKP mit der euphorischen Schlagzeile ,Sie haben es gewagt! " (138) und rief zur Solidaritätsbezeugung auf. Es folgten: ,Aktion gerechter Milchpreis" (139), „Weidinger Milchkrieg weitet sich aus" (140), „Nach dem Weidinger Milchkrieg“" (141), „Gefräßiger als ein Bär ${ }^{66}$ (142).

Der Tenor war kämpferisch: „,... aber das Diktat des Konzerns, der bisher selbstherrlich den Preis festsetzte, wurde gebrochen"(143). Zugleich mußte die DKP feststellen, daß es ihr nicht gelang, auf die Bewegung Einfluß zu nehmen. Thre Erklärung dafür ist typisch: „Aber die Bauern besuchten zwar die Versammlungen der DKP, übernahmen unsere Argumente, orientierten sich auch örtlich an der ,DKP-Landrevue', ließen sich aber doch von einigen Bauernverbandsführern und CSU-Fanatikern dazu bringen, sich gleichzeitig in einem ängstlichen Antikommunis-

135) Gerhard Müller, Ehrenfried Friedrich, Der Widerspruch zwischen Monopolisten und Bauernschaft und der Kampf der westdeutschen Bauern um ihre Existenz, Leipzig 1966, S. 19.

136) Ebenda, S. 18.

137) Ebenda, S. 19.

138) DKP-Land revue, Jahrgang 1, Nr. 3/71.

139) Ebenda, Jahrgang 1, Nr. 4/71.

140) Ebenda, Jahrgang 2, Nr. 1/72.

141) Ebenda, Jahrgang 2, Nr. 2/72.

142) Ebenda, Jahrgang 2 , Nr. $3 / 72$.

143) Ebenda, Jahrgang 2, Nr. 3/72. 
mus von den Kommunisten abzugrenzen." (144) Statt einer ökonomischen Analyse der Produktionsverhältnisse unter den speziellen Vertragsbedingungen der Alpenmilch AG, statt einer Untersuchung des politischen Gehaltes der Bewegung, wiederum die platte, politizistische Denunziation von Manipulateuren. Selbst hier, wo sich die Forderungen der Bauern direkt an ein Monopol wandten, ist die Einschätzung des Kampfes als eines antimonopolistischen oberflächlich und von Wunschvorstellungen geprägt. Der führende Kopf der Bewegung, der Fabrikant Wilhelm Neuner, der die "Aktion" ins Leben rief, das Startkapital vorschoß, als Sprecher der „Aktion" auftrat und schließlich wesentlich am Zustandekommen des Vergleichs beteiligt war, bezeichnete die DDR als ein Zuchthaus und sagte, auf ein Bündnis der "Aktion" mit der DKP hin angesprochen: „Die DKP hat also 30000 Mitglieder im Bundesgebiet. Das ist gar nichts. Allein in Bayern haben die Nervenheilanstalten 40000 Insassen. "(145) Die DKP-Landrevue hatte ihre Leser aufgefordert, an Neuner Solidaritätsbezeugungen zu schicken (146). Was die politische Orientierung der Bauern selbst betrifft, so kommt sie in ihren Forderungen zum Ausdruck: sie forderten einen gerechten Anteil, einen gerechten Preis. „Ausbeutung “ gebrauchten sie nicht im marxistischen Sinne, sondern eher als Synonym für "Übervorteilung". In der Tat handelte es sich bei der „Aktion" um eine Bewegung benachteiligter Kleinunternehmer mit eher vorkapitalistischem als antikapitalistischem Bewußtsein. Das kommt auch darin zum Ausdruck, daß 2000 Mitglieder der "Aktion“ das ,verbesserte November-Angebot" der Alpenmilch $A G$, das eine Erhöhung um ca 0,5 Pfennig $/ \mathrm{kg}$ brachte, annahmen, daß die Bauern benachbarter Gebiete mit höheren Milchpreisen die „Aktion“ nicht unterstützten, daß die verbleibenden 20000 DM des Kampffonds unter den Mitgliedern des „,inneren Komitees“ verteilt wurden und daß die Milcherzeugergemeinschaft, zu der sich ein Großteil der Weidinger Lieferanten nach der Aktion zusammenschloß, die Auseinandersetzung mit der Alpenmilch AG bislang nicht wieder aufgenommen hat.

Um auf die Eigentumsfrage zurückzukommen, ist schließlich zu bemerken, daß die Politik des Festhaltens am Bodeneigentum, wie sie die DKP betreibt, in der Bauernschaft mittlerweile keine unumstrittene Sache mehr ist. Auf einem Seminar des agrarpolitischen Arbeitskreises der Landjugend, das im Sommer 1971 in Stuttgart-Hohenheim stattfand, konnte man zu diesem Thema hören: „Wir sind doch nur noch Sklaven unseres Eigentums." Und Lambert schreibt im Zusammenhang mit der auch von der KPF vertretenen Wahlparole „Die Erde denen, die sie bearbeiten!“(147): „Aber die Mehrzahl der Bauern kann begreifen, daß dieser individuelle Besitz einer tiefen Entfremdung gleichkommt." (148)

Im folgenden einige weitere Aspekte der DKP-Bauernpolitik.

144) Ebenda, Jahrgang 2, Nx. 2/72.

145) Interview mit Wilhelm Neuner am 17.2. 1973.

146) DKP-Landrevue, Jahrgang 1 , Nr. $3 / 71$.

147) Mittlerweile hat die KPF unter dieser schon vom Kirchenvater Ambrosius aufgestellten Parole, die 1789 ihre Berechtigung hatte, eine Organisation zur Verteidigung des bäuerlichen Familienbetriebes ins Leben gerufen.

148) Lambert, a. a. O., S. 83. 


\section{Einschätzung der Bonner und EWG-Agrarpolitik}

Das Demokratische Bauernprogramm fordert: "Mit der bisherigen bavernfeind lichen EWG-Agrarpolitik ist Schluß zu machen", denn: „Der EWG-Agramarkt brachte den westdeutschen Bauern nur Verluste, Not und Sorgen, den Ver.

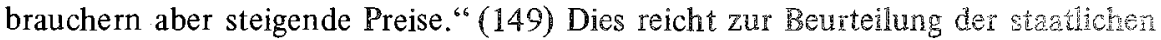
Agrarpolitik nicht aus. Scheringer/Sprenger und Rechtziegler befassen sicr ausfint" licher mit der Wandlung vom Leitbild des „,bäuerlichen Familienbetriebes ${ }^{66}$ zur Politik der „Gesundschrumpfung66, zur ,wachstumsorientierten Agrarpolitik", einer Agrarpolitik, die die Forderung erfullen soll, mit ,der wirtschaftspolitischen Grundlinie der Industrie (überein) zustimmen." (150) Einerseits stimmt $€ s, d 26$ die Ideologie des bäuerlichen Familienbetriebes eine wichtige Funktion in der antikommunistischen Propaganda hat (, Heute handelt es sich um die Erhaltung und die Vermehrung selbstständiger Existenzen als Gegengewicht gegen den Kollekivisu mus und Kommunismus." (151)), und es stimmt auch, wenn Rechtziegier schreibt, daß die Theorie des Familienbetriebes, in ihrem Wesen demagogisch is $t^{66}$ und die hauptsächlich über die Vertragslandwirtschaft stattfindende Konzentration in der Landwirtschaft verschleiert (152). Andererseits ist aber ein modifizierter Familienbetrieb Kernstück der neuen Agrarpolitik. Es soll lediglich das, „was unter einem Familienbetrieb verstanden wird, im Zuge der technischen Entwicklung immer weiter nach oben" (153) verschoben werden. Die von Mansholt propagierten „modernen Landwirtschaftsunternehmen" und „Produktionseinheiten" sind für die vertikale und horizontale kapitalistische Integration zurechtgemachte Familienbetriebe. Die von ihm vorgeschlagenen Betriebsgrößen, 80-120 ha für Getreide- und Hackfruchtbetriebe, lassen sich von einer Familie bewirtschaften, wie die Entwicklung in den USA zeigt, wo die Durchschnittsgröße der Farmen 1964 bei 133 ha lag. Die Vergrößerung des Familienbetriebes wird nicht durch die technischen Möglichkeiten beschränkt, sondern durch die bundesrepublikanische Agrarstruktur. In der gegenwärtigen Phase der kapitalistischen Durchdringung der Landwirtschaft sucht die Bonner und EWG-Agrarpolitik einen Kompromiß zwischen der aws politischen Gründen (Vermeidung von sozialen Härten und Untuhe) langsam voranschreitenden Veränderung der Agrarstruktur, dem Stand der wissenschaflichtechnischen Entwicklung und einer möglichst billigen Agrarproduktion. Im Kapitalismus erlaubt die Familienarbeitsverfassung unter Arpassung an die sich langsam wandelnde Agrarstruktur die kostengüstigste Produktion landwirtschaflicher Erzeugnisse (gewährleistet auch durch die sich pauperisierenden Betriebe, die meist ohne Verzinsung des Kapitals und weit unter dem gewerblichen Vergleichslohn wirtschaften). Niedrige Erzeugerpreise bedeuten aber auch guite Profitmöglichkeiten

149) Demokratisches Bauernprogramm der DKP, a. a. O., S. 24

150) Jahresbericht des BDI, zitiert bei Scheringer/Sprenger, a. a. O., S. 125.

151) Hüttebräucker, Agrarstruktur, ein politisches, soziologisches oder ökonomisches Problem. Zitiert bei Scheringer/Sprenger, a. a. O., S. 120.

152) E. Rechtziegler, Neue Entwicklungstendenzen ..., a. a. O., S. 152.

153) Ebenda, S. 153. 
für die Verarbeitungsindustrie und den Handel und immer noch relativ niedrige Verbraucherpreise, also niedrige Kosten der Reproduktion der Ware Arbeitskraft. Es wird also angestrebt, daß sich die optimale Betriebsgröße jeweils auf einen Familienbetrieb mit weitestgehender Ausnutzung dẹ gegebenen technischen Möglichkeiten einpegelt, wobei der Parameter Agrarstruktur durch die Schaffung neuer landwirtschaftlicher Unternehmensformen und flankierende Maßnahmen der Boden-, Markt- und Sozialpolitik langsam in das den natürlichen Gegebenheiten entsprechende Optimum gebracht wird.

$\mathrm{Zu}$ dieser Tendenz der Modernisierung des bäuerlichen Familienbetriebes ist die Erhaltung des bäuerlichen Eigentums zweifellos keine Alternative. Ebenso ist es eine Illusion, über die Systemkonkurrenz, (was mit der Forderung nach ,Schaffung eines europäischen Sicherheitssystems (mit) normalen Handels- und Wirtschaftsbeziehungen zu allen europäischen Staaten" (154) angedeutet wird) das Vordringen des Kapitals in der Landwirtschaft aufhalten zu wollen. Und die Forderung schließlich, daß ,der Ausgleich der Verluste durch die EWG und die DM-Aufwertung... grundsätzlich so geregelt werden (muß), daß jeder geschädigte Bauer den Verlust auf Mark und Pfennig ersetzt bekommt ${ }^{66}(155)$, geht von der irrigen Annahme aus,

- der Nationalstaat könne durch bloße antimonopolistische Umfunktionierung die Folgen der Weltagrar- und Weltwährungskrise von der Landwirtschaft fernhalten,

- die kapitalistische Integration der Agrarmärkte innerhalb der EG könne die Anpassung der Landwirtschaft an die wirtschaftliche Grundlinie der Industrie in eine bauernfreundliche Agrarpolitik verwandeln.

\section{Einschätzung der Genossenschaften}

Im DKP-Bauernprogramm findet sich neben der richtigen Forderung ,Die Umwandlung des genossenschaftlichen Eigentums in Aktiengesellschaften ist strikt abzulehnen ..."(156) der seltsame Satz: „Anliegen des ländlichen Genossenschaftswesens muß es sein, im Geiste der Prinzipien Raiffeisens vor allem die gegenseitige Hilfe für die kleinen und wirtschaftlich schwächeren Bauern zu organisieren." (157) (Hervorhebung v. V.) Marx, ein Zeitgenosse von FriedrichWilhelm Raiffeisen (Raiffeisen gründete im Notwinter 1846/47 im Westerwald die ersten genossenschaftlichen Hilfsvereine) zeigte bereits, daß die Kooperation im Kapitalismus nicht in der Lage ist, das Wachstum der Monopole aufzuhalten, das Elend der Massen zu erleichtern. Marx schrieb, daß das „Kooperativsystem... nicht im Stande ist, durch sich selbst die kapitalistische Gesellschaft umzugestalten. "(158) Lenin schrieb in „Über das Genossenschaftswesen“, daß die Pläne der

154) Demokratisches Bauerprogramm der DKP, a. a. O., S. 24.

155) Ebenda, S. 25.

156) Ebenda, S. 32.

157) Ebenda, S. 33.

158) Marx, Instruktionen an die Delegierten des provisorischen Zentralkomitees über einzelne Fragen. In: Der Vorbote, 1866, Nr. 10, S. 153. Vgl. ders., Instruktionen. für die .Delegierten des Provisorischen Zentralrats ...., Werke Bd. 16, Berlin 1964, S. 195. 
alten Genossenschaftler zur Umwandlung der kapitalistischen Gesellschaft auf friedlichem Wege durch die Genossenschaften falsch waren, weil sie den politischen Kampf zum Sturz der Kapitalistenklasse negierten (159). Darin kommt zum Ausdruck, daß das soziale Wesen der Genossenschaften von der jeweils herrschenden Gesellschaftsordnung und der politischen Macht bestimmt wird. Und Bucharin: „Mit den Mitteln der Kooperation und der Genossenschaft erhalten sich nun die Bourgeoisie und Großagrarier ihren Einfluß auf die Bauernschaft.“(160) Rechtziegler beurteilt die Raiffeisengenossenschaften ebenfalls negativ: sie sind ,gegenwärtig weder imstande noch willens, die wirtschaftlichen und politischen Machtpositionen des Monopolkapitals brechen zu helfen." und: „Gegenwärtig unterstützen sie jedoch die Organisation einer monopolkapitalistischen vertikalen Kooperation... Sie wollen das Vertrauen der Bauern zu den Genossenschaften für die Organisierung der vertikalen Kooperation im Interesse des Monopolkapitals ausnutzen." (161) Lambert nennt die Kooperation schließlich eine ,Schleuse zwischen den kleinen Produzenten und den Großkapitalisten“, ,Transmissionsriemen des Kapitalismus“, und konstatiert: „Die Genossenschaft hat also nicht die Möglichkeit, die Natur der Wirtschaftsordnung zu verändern. Um zu überleben, wird sie gezwungen sein, sich die kapitalistischen Mechanismen zu eigen zu machen..."(162)

Diese Einschätzung der landwirtschaftlichen Genossenschaften wird voll bestätigt, wenn man den „Geist der Prinzipien Raiffeisens ${ }^{66}$ etwas näher betrachtet: Raiffeisen selbst hat den Zweck seiner Genossenschaften so beschrieben: „Die liebevolle Fürsorge der besitzenden Klassen für die Ärmeren, in der innigen Vereinigung, wie solche die in Rede stehenden Genossenschaften bieten, ist das einzige Mittel, die rechte Innung der Zukunft zu begründen und der Umsturzpartei den Boden für ihre verderbliche Agitation zu entziehen. Das ist christlicher Sozialismus!" (163)

- Hugo Tillmann, Lehrbeauftragter für Genossenschaftswesen an der Freien Universität Berlin, schrieb zur Einführung von Raiffeisens Buch über die Prinzipien Raiffeisens im Gegensatz zu denen von Marx: „Weit entfernt davon, die Bedürftigen gegen die Besitzenden zu sammeln, gab er den Anstoß von oben her: die Vermögenden bringen zunächst die Mittel zusammen, verbürgen sich mit ihrem Besitz, stellen ihre Arbeitskraft, ihr Ansehen, ihren Kredit in den Dienst der Sanierung der vom Unglück heimgesuchten, der sozial Schwachen, zugleich als Ermutigung für deren Selbsthilfe. Darin liegt das Aufbauende und Versöhnende des Raiffeisentums; es zielt auf die Wiederherstellung des sozialen Gleichgewichtes und der Dorfgemeinschaft." (164)

159) Lenin, Ausgewählte Werke in zwei Bänden, Bd. II, S. 1010.

160) Bucharin, a. a. O., S. 20.

161) Rechtziegler, Westdeutsche Landwirtschaft ..., a. a. O., S. 79/80, S. 89.

162) Lambert, a. a. O., S. 38, S. 85, S. 52.

163) F. W. Raiffeisen, Die Darlehenskassen-Vereine, 7. Aufl., Verlag der Raiffeisen-Druckerei GmbH. Neuwied a. Rh., 1950, S. 35, 36.

164) In: Raiffeisen, $a_{\circ} a_{0} O ., S . X I$. 
- Raiffeisen war kein Antisemit; den Wucher, den er mit seinen Genossenschaften bekämpfte, erkannte er durchaus als ein bei Juden und Christen gleichermaßen verbreitetes „Blutsaugergeschäft". Gleichwohl erleichterten seine paternalistische Grundeinstellung und sein Streben nach Vereinigung ,aller Stände und aller Glieder des deutschen Volkes“" in ,christlicher Solidarität" (165) die Selbstgleichschaltung der landwirtschaftlichen Genossenschaften im Faschismus. Im ,Deutschen Landwirtschaftlichen Genossenschaftsblatt“ vom 30. April 1933 heißt es denn auch: „Die landwirtschaftlichen Genossenschaften sind in eine neue Phase ihrer Entwicklung eingetreten, der sie sich nicht verschließen konnten und auch nicht wollten. In einer Zeit, die dem deutschen Bauern wieder neuen Mut in seiner schweren Arbeit gegeben hat,... werden sicherlich auch diese wichtigsten Glieder der landwirtschaftlichen Selbsthilfe mit höchster Kraftentfaltung sich einsetzen und nach Abstoßung mancher in den Irrnissen und Wirrnissen der letzten Jahrzehnte erworbenen Schlacken sich des großen Erbes eines Raiffeisen, eines Wilhelm Haas wieder voll und ganz würdig erweisen. Nichts Wesensfremdes tritt in ihren Gedanken- und Arbeitskreis, war doch genossenschaftliches Wirken, dort, wo es echt und wahr war, immer national, immer schon dem Geiste nach - nationalsozialistisch." (166)

- Wie die „Existenzbedingungen der Raiffeisen-Genossenschaften im Kapitalismus" nicht durch den Kampf der Bauern, sondern im Geiste Raiffeisens verbessert werden, beschreibt Hans Lukas im Zusammenhang mit der Integration zwischen dem Deutschen Raiffeisenverband und dem Deutschen Genossenschaftsverband: „Durch die Beseitigung des von Anfang an bestehenden und in den letzten Jahren immer heftiger werdenden Konkurrenzkampfes wird eine geschlossene Organisation entstehen, die sich im Wettbewerb mit anderen Unternehmungen behaupten kann. Damit hat sich der von Raiffeisen und Schulze-Delitsch postulierte Selbsthilfegedanke von neuem bestätigt." (168)

Während Rechtziegler eine Nutzbarmachung der Genossenschaften für den Kampf der Bauern von ihrer großzügigen staatlichen Förderung abhängig macht und die DKP ihren Blick nach rückwärts zu den Prinzipien Raiffeisens wendet, diskutiert Lambert die Möglichkeit der Zusammenarbeit von Genossenschaftsarbeitern und -angestellten und Bauern sowie die Frage, ob die proletarisierten Bauern die Verwaltung der Genossenschaften übernehmen können. Er stellt die Frage klar in den

165) Ebenda, S. 6

166) Zitiert bei: Kuno Blundau, Nationalsozialismus und Genossenschaften. Schriftenreihe des Forschungsinstituts der Friedrich-Ebert-Stiftung. Verlag für Literatur und Zeitgeschehen, Hannover 1968, S. 71, 72.

168) Hans Lukas, Der Deutsche Raiffeisenverband. Entwicklung, Struktur und Funktion. Untersuchungen über Gruppen und Verbände, Band 11, Verlag Duncker und Humboldt, Berlin 1972, S. 162. 
Rahmen des Kampfes „gegen die Illusion der bürgerlichen Kooperation“ (die Raiffeisen-lllusion) und für die „Eroberung des Apparates“ durch die Bauern ,in Verbindung oder gemeinsam mit den Arbeitern" (169).

\section{Einschätzung des Deutschen Bauernverbandes (DBV)}

Das Demokratische Bauernprogramm erwähnt den DBV oder eine alternative Vertretung der Bauern nicht. Scheringer/Sprenger formulieren folgende Marschroute: „Das Ziel sollte sein, daß der Bauernverband, in dem etwa 90 Prozent aller westdeutschen Bauern organisiert sind, eine ebensolche Kampforganisation gegen das Monopolkapital wird, wie das die fortschrittlichen Gewerkschaften für die Arbeiterklasse sind."(170) Rechtziegler schreibt, daß die DBV-Politik die gesellschaftliche Isolierung der Bauern bewirkt hat. „Die politische Plattform der Bauernverbandsführung erweist sich daher als bauernfeindlich." Der Verband kann aber nach seiner Meinung, im Interesse der Bauern ... wirksam werden, wenn er die Politik ... der Kompromisse mit dem Monopolkapital aufgibt und durch seine aktive Zusammenarbeit mit der Arbeiterklasse ersetzt, ... zu einem politischen Kampfinstrument ausgebaut wird ...“(171). Hier begegnen wir einer ähnlichen, im Grunde voluntaristischen Einschätzung, wie sie die DKP auch zur Erklärung der pro-kapitalistischen Politik der SPD heranzieht: die Grenzen einer antimonopolistischen Politik des Bauernverbandes liegen lediglich in den gegenwärtigen Machtverhältnissen innerhalb des Verbandes, in seiner Politik der Klassenzusammenarbeit (analog zur Politik der, ,rechten Führer der SPD“).

$\mathrm{Ob}$ politisches oder gewerkschaftliches Kampfinstrument, beide zitierten Auffassungen gehen im Grunde von der Annahme aus, daß der Bauernverband ein Klassenorgan der gesamten Bauernschaft ist oder zu einem solchen gemacht werden kann. $\mathrm{Zu}$ diesem Schluß muß man kommen, wenn man die Klassengegensätze innerhalb der Bauernschaft, die sich politisch in der Beherrschung des Bauernverbandes durch die Großbauern ausdrücken, vernachlässigt. (Andererseits erübrigt sich dann auch die Frage, weshalb der DBV trotzdem einen Massenanhang unter den Kleinbauern hat, mit welchen Mitteln die Partnerschaftsideologie hier aufrechterhalten wird.) Ein Vergleich mit den Gewerkschaften ist jedenfalls, klassenmäßig betrachtet, unzulässig, denn die Gewerkschaften sind Klassenorgane der Arbeiter, der DBV aber ist ein Klassenorgan der Großbauern und damit der Bourgeoisie. In der sich regenden Kritik einiger Bauerngruppen am DBV einerseits und den Anstrengungen der Verbandsleitung andererseits, die spontanen Aktionen der Bauern unter Kontrolle zu halten und in von ihr organisierten Demonstrationen zu kanalisieren, kommen die Klassengegensätze innerhalb der Bauernschaft zum Ausdruck. Für den Kampf zwischen Bourgeoisie und Arbeiterklasse um die Unterstützung der Bauern gilt noch heute, was Bucharin 1925 feststellen mußte:

169) Lambert, a. a. O., S. 83 bis 89.

170) Scheringer/Sprenger, a. a. O., S. 163.

171) Rechtziegler, Westdeutsche Landwirtschaft ..., a. a. O., S. 106. 
„Wir sprechen über Einheitsfronttaktik, wir haben über die Parole der Arbeiter- und Bauernregierung gesprochen, wir haben Resolutionen über die Notwendigkeit der Eroberung der Bauernschaft angenommen usw., aber wir tun praktisch sehr wenig auf diesem Gebiet, während die Bourgeoisie die wirkliche Einheitsfronttaktik im Verhältnis zu den Bauern anwendet, sehr viel auf diesem Gebiet leistet, sehr große Erfolge erzielt und eine Reserve gegenüber dem Proletariat formiert." (172) Als die Macht im Staat hat die Bourgeoisie die besseren Instrumente: heute wie damals unter anderem das ländliche Kredit- und Genossenschaftswesen und die außenwirtschaftliche Absicherung des Agrarmarktes. Ihr Klassenorgan in der Bauernschaft war damals der „Reichslandbund“ (in dem die Hälfte der Landarbeiter organisiert war!), ist heute der DBV. Es wäre schön, wenn die Großbourgeoisie nur ,gemeinsame Interessen ... mit den einfachen Warenproduzenten (Bauern, Handwerker), den Einzelhändlern, sowie der kleinen und mittleren Bourgeoisie zu konstruieren" sich bemühte (173). (Hervorhebung v. V.) Die Politik des DBV zeigt hingegen die tatsächliche Interessengemeinschaft: der DBV-Präsident Constantin Freiherr Heereman von Zuydtwyck hat das in seiner ,neuen Politik" klar formuliert, wenn er sagt, „Interessenverbände wie der BDI und DBV ... sind notwendig, um im Rahmen zunehmender staatlicher Einflußnahme eine demokratische ,Mitbestimmung" der Betroffenen, der Unternehmer, zu gewährleisten." (174) Was von einer technokratischen Ausrichtung der DBV-Politik zu erwarten ist, zeigt die Entwicklung in Frankreich. Lambert schreibt über die Politik der CNJA (Conseii National de la Jeunesse Agricole = Nationalverband der jungen Landwirte): „Es handelt sich um intellektuellen und technokratischen Paternalismus. Er erzeugt Unterstützungsempfänger." (175)

Die bisher behandelten Einschätzungen und Forderungen aus dem DKPBauernprogramm verdeutlichen in etwa die Tendenzen der DKP-Bauernpolitik. Der gesamte Katalog der Forderungen soll hier nicht im einzelnen diskutiert werden. Es sind, wie nicht anders zu erwarten, fast durchwegs Forderungen an den Staat. Zum Teil decken sie sich mit Forderungen des DBV (z. B. „Die Erzeugerpreise müssen stabil sein, die Produktionskosten decken und den Bauern eine Rendite garantieren." (176), zum Teil mit Forderungen bürgerlicher Kultur- und Sozialpolitiker, die teilweise auch schon vom Staat aufgegriffen wurden (,Anpassung der landwirtschaftlichen Ausbildung an die Erfordernisse der wissenschaftlichtechnischen Revolution“, „Fortbildungs- und kulturelle Veranstaltungen“, „, die Einführung einer gesetzlichen Krankenversicherung und die Verbesserung des Unfallversicherungsschutzes“, „eine ausreichende Altersversorgung", „Mutter- und Kinderschutz“, „Ganztags-Kindergärten“ (177)), zum Teil sind es Floskeln (,eine

172) Bucharin, a. a. O., S. 16

173) Scheringer/Sprenger, a. a. O., S. 152.

174) Rede vor der Mitgliederversammlung des Bundesverbandes der Deutschen Industrie (BDI) in Hamburg am 1. 7. 70. Zitiert bei: Poppinga, Zur Agrarfrage in der Bundesrepublik, in: Lambert, a. a. O., S. 139.

175) Lambert, a. a. O., S. 64.

176) Demokratisches Bauernprogramm der DKP, a. a. O., S. 27.

177) Ebenda, S. 35, 36, 38, 39. 
neue, fortschrittliche Bildung, getragen vom humanistischen Geist", ,ein vielfältiges, kulturvolles Leben“( 178$)$ ).

An dieser Stelle sollen nur noch zwei Beispiele angeführt werden, die gewisse Widersprüchlichkeiten innerhalb derselben politischen Grundauffassung verdeutlichen.

\section{Zur Pacht}

Im Demokratischen Bauernprogramm heißt es dazu: „Die Pachtverhältinisse sind neu zu regeln. Der Pachtschutz ist wieder einzuführen. (Siehe Reichspachtschutzordnung vom 30. Juli 1940, RGB1. 1, S. 1065 - Anmerkung v. V.) Bäuerlichen Pächtern sind Pachtzeiten von 15 bis 25 Jahren einzuräumen." (179) (Hervorhebung v. V.) Was für den bäuerlichen Pächter gut ist, muß es nicht auch für den bäuerlichen Verpächter (z. B. heute den Arbeiterbauern) sein. Rechtziegler stellt fest: „Die Bundesregierung will die Vergrößerung der ,förderungswürdigen“ Betriebe in Zukunft vor allem auf dem Wege der Zupacht begünstigen..." Aber ,die Neigung der ruinierten Bauern, ihren Boden langfristig zu verpachten, ist bisher, trotz aller staatlichen Förderung, gering. Denn der Abschluß eines langfristigen Pachtvertrages, ,der wirtschaftlich einer Veräußerung sehr nahe kommt ${ }^{6}$, wie der frühere Landwirtschaftsminister Höcherl zugeben mußte, entspricht keineswegs den Interessen der kleinen Verpächter. Sie möchten auch künftig mehr oder weniger kurzfristig über ihren Boden verfügen können." (180) (Hervorhebung v. V.)

\section{Erzeuger- und Produktionsgemeinschaften}

Das DKP-Bauernprogramm fordert ,echte zwischenbetriebliche und gemeinschaftliche Zusammenarbeit" und „eine breite Entfaltung der gegenseitigen Hilfe, der zwischenbetrieblichen Zusammenarbeit ... in demokratischen Erzeuger- und Produktionsgemeinschaften."(181) Sowohl Scheringer/Sprenger als auch Rechtziegler gehen ausfuihrlich auf den ,Entwicklungsstand der horizontalen Koopera-

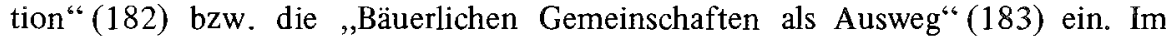
Gegensatz zu Scheringer/Sprenger betont Rechtziegler, daß die Maschinenringe, bei denen die Maschinen Eigentum der eirızelnen Bauern bleiben und die nach dem Gewinnprinzip arbeiten, im Vergleich zu den Maschinengemeinschaften, die nach dem Selbstkostenprinzip arbeiten, ,von vorneherein das Entstehen von gemeinschaftlichem Eigentum verhindern." Ebenso skeptisch ist Rechtziegler gegenüber den Erzeugergemeinschaften: „Das westdeutsche Monopolkapital erblickt in den Erzeugergemeinschaften ein Instrument, um mit Unterstützung der Bauern ein ihren Wünschen entsprechendes Angebot von Waren zu erhalten, ohne daß sich die Marktstellung der Bauern grundsätzlich verbessert. Die Förderung der Erzeugergemeinschaften durch staatliche Mittel verfolgt den Zweck, ihre Entwicklung in

178) Ebenda, S. 35.

1.79) Ebenda, S. 19.

180) Rechtziegler, Westdeutsche Landwirtschaft ..., a. a. O., S. 45, 46.

181) Demokratisches Bauernprogramm der DKP, a. a. O., S. 21.

182) Rechtziegler, Westdeutsche Landwirtschaft ..., a. a. O., S. 30-57.

183) Scheringer/Sprenger, a. a. O., S. 102-118. 
eine Richtung zu drängen, die den Kapitalverwertungsinteressen der Monopole entspricht. Die gegenwärtig gültigen Bonner Richtlinien sehen eine Förderung der Erzeugergemeinschaften in den ersten drei Jahren ihrer Existenz durch degressive Beihilfen nur dann vor, wenn sie von den obersten Landesbehörden anerkannt werden und sich einer Reihe diskriminierender Bestimmungen unterwerfen. Damit wird das Ziel verfolgt, die Entwicklung der Erzeugergemeinschaften unter Kontrolle zu halten und sie zu einem das staatsmonopolistische System stabilisierenden Faktor zu entwickeln."(184) Scheringer/Sprenger interpretieren dagegen die Erzeugergemeinschaften als Errungenschaften der Bauern: „Die Bauern versuchen dadurch, ihre ökonomische Kraft wenigstens auf dem Markt zu vereinigen, um dem Druck der kapitalistischen Verarbeitungs- und Handelsbetriebe besser standhalten zu können und den Anforderungen des Marktes besser zu entsprechen."(185) (Hervorhebungen v. V.)

Sowohl Rechtziegler als auch Scheringer/Sprenger sehen, daß die Produktionsgemeinschaften in der BRD ,kapitalistischen Gesetzmäßigkeiten unterliegen, da sie in das herrschende kapitalistische System eingeordnet sind" (186), und daß ,über den Charakter der horizontalen Kooperationsgemeinschaften . . . der Klassenkampf zwischen Monopolen und Bauernschaft entscheidet.“(187) Rechtziegler scheint aber den Vorsprung der Bourgeoisie auch auf diesem Gebiet realistischer einzuschätzen: „In den letzten Jahren hat das Monopolkapital seine Anstrengungen verstärkt, um die Bauern von vorneherein auf solche Formen der Kooperation zu orientieren, die sich in das staatsmonopolistische Herrschaftssystem einfügen." (188)

Nur wenn die Ambivalenz von Reformen innerhalb des Kapitalismus richtig erkannt wird, kann der positive Aspekt von bäuerlichen Produktionszusammenschlüssen richtig bewertet werden. Scheringer/Sprenger schreiben dazu: ,In bestimmtem Umfang wird die eingefahrene individuelle Arbeits- und Lebensweise verändert“(189), und Lambert hebt - wenn gleichzeitig „die Tendenzen zum kollektiven Kapitalismus“ bekämpft werden - die „Bildung eines kollektiven Bewußtseins... aus der täglichen Praxis" hervor; "auf dieser Ebene ist die Gruppenlandwirtschaft wichtig." (190a)

Schließlich noch einige Bemerkungen zur Agitation der DKP in der Bauemfrage $(190 \mathrm{~b})$

Hauptinstrument der Agitation auf dem Lande ist die DKP-Landrevue. Nicht von ungefähr besteht das Kernstück jeder Ausgabe, das auch graphisch hervorgehoben wird, aus einer Reportage über die sozialistische Landwirtschaft in der DDR.

184) Rechtziegler, Westdeutsche Landwirtschaft ..., a. a. O., S. 51, 52.

185) Scheringer/Sprenger, a. a. O., S. 108.

186) Ebenda, S. 114.

187) Ebenda, S. 115.

188) Rechtziegler, Westdeutsche Landwirtschaft .... a. a. O., S. 54.

189) Scheringer/Sprenger, a. a. O., S. 118.

190a) Lambert, a. a. O., S. 19.

190b) Der folgende Abschnitt kann nur einige Hinweise geben. Für eine ausführlichere Anlayse müßte die Landrevue und insbesondere auch die UZ im einzelnen untersucht werden. 
Einerseits sind das wichtige Beiträge zur Bekämpfung des Antikommunismus und der falschen, vor allem von der landwirtschaftlichen Presse geprägten Vorstellungen über die DDR-Landwirtschaft, andererseits wird mit dem starken Herausstreichen der technischen Errungenschaften ein wenig Bauernfängerei betrieben. In fast keiner Reportage fehlen Bilder von PS-starken Großmaschinen, und welcher Bauer sähe sich nicht gerne als ,Herr ${ }^{66}$ über ein halbes Dutzend Getreidefelder durchpflügende Mähdrescher!

Wichtig für die Propagierung des Arbeiter-Bauern-Bündnisses ist der Kampf gegen die latente Arbeiterfeindlichkeit vieler Bauern und gegen solche, auf dem Lande besonders festsitzenden Märchen wie das von der Lohn-Preis-Spirale. In dieser Hinsicht liefert die Landrevue gute Beiträge: z. B. über die ChemiearbeiterStreiks (in Nr. 3/71), „Es gibt nur die Preis-Profit-Spirale“ (in Nr. 4/71), „Die Bauern zahlen kräftig mit" (in Nr. 1/71, über die BASF). Im Gegensatz dazu kann man in der Haupt-,,Zeitung der DKP', der UZ (Unsere Zeit) nur wenig über die Probleme auf dem Lande lesen. In der Zeit von Mitte April bis Mitte Juni 1972 wurde beispielsweise lediglich über Bleivergiftung von Kühen durch Bleistaub der Preussag-Weser-Zink GmbH (26. 5. 72 und 16. 6. 72, eher einer der zahlreichen Beiträge zur Frage des Umweltschutzes) und über Proteste von Bauern gegen ein Nato-Manöver bei Nürnberg (9. 6. 72) berichtet und ein ,Interview mit dem Vorsitzenden einer landwirtschaftlichen Produktionsgemeinschaft in der DDR“ gebracht (26. 5. 72), das auf die Probleme der BRD-Landwirtschaft nicht eingeht. Auch im Mai-Aufruf der DKP 1972 bleiben die Bauern unerwähnt: „Die Zurückdrängung und Überwindung der Herrschaft der Monopole können nur das Werk der kämpfenden Arbeiter und Angestellten selbst und ihrer Gewerkschaften sein.“"(UZ, 20. 4. 72)

Zur Einbeziehung der Großbauern in die Bauern-Einheitsfront wird gern der 60-ha-Bauer Heinrich Biester zitiert: „In unserer Partei, als politische Organisation, können auch alle Klein- und Mittelbauern, auch diejenigen, die heute von uns noch abseits stehen, der größte Teil auch der gegen uns gerichteten, sogenannten Großbauern, eine Plattform im Kampf gegen die Ausbeutung des Großkapitals finden." (191)

Die Wirkung der DKP-Arbeit unter den Bauern wird in folgender Geschichte variiert: Richard Scheringer (192) erzählt: „Die Bauern hatten sich mehr versprochen. In Zügen und Omnibussen erinnert sich mancher, daß etwas verteilt worden war während der Kundgebung: Das Demokratische Bauernprogramm der Deutschen Kommunistischen Partei. Hier und dort zieht es einer aus der Tasche und studiert." Und Hermann Sittner (193): „Und in allen Omnibussen, die die Kundgebungsteilnehmer wieder nach Hause führen, sah man Bauern die ,DKP-Landrevue" lesen ..."

191) Arbeiter und Bauern - gemeinsam für demokratischen Fortschritt. Agrarpolitische Konferenz der DKP, 21. Februar 1970, Frankfurt a. M., S. 29, 30; und Scheringer/ Sprenger, a. a. O., S. 160.

192) DKP-Landrevue, Jahrgang 1, Nr. 2/71.

193) Ebenda, Jahrgang 1, Nr. 4/71. 
Der Hinweis auf Flicks Stundenlohn darf auch in der Landrevue nicht fehlen: 218844 Mark (194). In derselben Ausgabe wird im Stil einer Agitation, die sich eher gegen die „Superreichen“ als gegen den Kapitalisten als „ökonomische Charaktermaske" und das Kapital als ein alles beherrschendes Produktions- und Gesellschaftsverhältnis richtet, darüber informiert, daß die Zahl der Millionäre in NordrheinWestfalen von 1966 bis 1969 ,um mehr als 1000 gewachsen ist" (195).

Das antimonopolistische Bündnis aller demokratischen Kräfte erlaubt es, den Klassenbegriff Arbeiter in einem Zug mit der klassenmäßig nicht faßbaren Kategorie Jugend zu nennen; z. B.: „Arbeiter, Bauern und Jugend gemeinsam gegen Großkapital."(196) Die Bauern werden aber normalerweise nicht gesondert aufgefuihrt: „Nach der Ratifizierung der Verträge sind wir veranlaßt, - ... die Frage zu beantworten, wie es jetzt weitergehen soll. Darauf wollen die Arbeiter, will die Jugend, will die ganze Partei eine Antwort."(197)

\section{Zusammenfassung: DKP-Bauernpolitik als integraler Bestandteil der DKP-Politik}

In der Einschätzung der Bauernfrage durch die DKP findet sich die der Stamokap-Theorie eigene Verwischung der Klassengegensätze auf zwei Ebenen wieder:

- Die mannigfaltigen Klassenwidersprüche in unserer spätkapitalistischen Gesellschaft werden dem Widerspruch zwischen Volk und Monopolen untergeordnet. Analog etwa zur Politik der ",gewerkschaftlichen Orientierung" des MSB Spartakus, die von der Gleichgerichtetheit der Interessen von Proletariat und Intelligenz ausgeht, wo es sich lediglich um die Tendenz handelt, daß Teile der Intelligenz sich an das Proletariat annähern, werden in der Bauernpolitik die ständischen Interessen, die auf eine Verteidigung des Eigentumsprivilegs gegenüber dem Proletariat hinauslaufen, in fortschrittliche, antimonopolistische Interessen umgemünzt.

Die Theorie des Stamokap erhebt den Systemgegensatz zwischen Kapitalismus und Sozialismus über die Klassengegensätze in den kapitalistischen bzw. sozialistischen Gesellschaften. Das Hauptziel des Kapitalismus wird im Zusammenschluß aller Kräfte gegen den Sozialismus gesehen, wenn etwa Rechtziegler schreibt: ,Für den westdeutschen Imperialismus ist insbesondere eine Neuorientierung seiner Agrarpolitik notwendig geworden, weil die sozialistische DDR demonstriert, wie die ökonomischen und sozialen Probleme der technischen Revolution in der Landwirtschaft erfolgreich gemeistert werden können." (198)

194) Ebenda, Jahrgang 2, Nr. $1 / 72$

195) Ebenda, Jahrgang 2, Nr. $1 / 72$.

196) Ebenda.

197) Kurt Bachmann, Zur Einschätzung der politischen Lage und zu den nächsten Aufgaben im Kampf für die Verwirklichung der Verträge von Moskau und Warschau, für Frieden und Sicherheit in Europa. Referat auf der 3. Tagung des Parteivorstandes der DKP. UZ-Extra, Eigenbeilage Nr. 22/72, S. 9.

198) Rechtziegler, Westdeutsche Landwirtschaft ..., a. a. O., S. 35. 
POLITLADENDRUCKE

Politladen-Druck 4

Christel Neusüss, Berlin

IMPERIALISMUS UND

WELTMARK TBEWEGUNG

DES KAPITALS

256 Seiten, DM 9,00
Politladen-Druck 5

Karl Held, Müchen

KRITIK DER

SPRACHWISSENSCHAFT

Erscheint im 1YQuartal '73!

Ca. 370 Seiten, ca. DM 14,00

Politaden-Drucke 1.7 uad Politiden-Reprints $1-18$ gibt es in allen linken und vlelen bürgerlichen Buchbandlungen und durch die Post bel Direktbestellunt an den

POLITLADEN ERLANGEN 852 ERLANGEN, HINDENBURGSTRASSE 17

Unsere Buchversandabteilung

MARXISTISCHE LITERATUR

PER POST

sendet Ihnen unsere eigenen Titel und alle in dieser "Prokla' annoncierten Bücher und Broschiren portofrei auf Rechnung zu.

Kataloge und Versandlisten anfordern beim POLITLADEN 852 ERLAIGEN W.V.SIEMENS-STR. 8

POLITLADENREPRINTS

NEU

Politladen-Reprint 17

DIE

KOMMUNISTISCHE

INTERNATIONALE

$1919 \cdot 1941$

37.900 Seiten in ca. 40 Bänden.

Kartoniert, klebegebunden.

Politladen-Reprint 18

Martov/Dan

GESCHICHTE DER RUSSISCHEN SOZIALDEMOKRATIE

Berlin 1926

Mit einem Vorwort von Hartmut Mehringer.

348 Seiten, 10,00 DM.

Sonderprospekt anfordern beim Verlag:

POLITLADEN ERLANGEN 852 ERLANGEN, HINDENBURGSTR. 
Dabei wird ignoriert,

daß sich der Kapitalismus immer noch primär aufgrund seiner immanenten Widersprüche bewegt,

- daß die Systemkonkurrenz keineswegs nur eine einseitige Beeinflussung des Kapitalismus durch den Sozialismus bedeutet.

Diese Bestandteile der Stamokap-Theorie können ihre historischen Wurzeln nicht verleugnen: die Subsumption der nationalen Klassenkämpfe unter die Systemkonkurrenz geht unmittelbar auf die stalinistische These vom Aufbau des Sozialismus in einem Lande zurück, und das breite antimonopolistische Bündnis aller demokratischen Volkskräfte ist nichts anderes als die Neuauflage der Volksfrontstrategie, die aus einer Defensivstrategie gegen den Faschismus in eine Strategie zur Verteidigung und Stärkung des ,sozialistischen Lagers" ${ }^{\text {" }}$ verwandelt wurde, weil der Theorie zufolge die Chancen eines ,friedlichen Übergangs zum Sozialismus" mit dem militärpolitischen Machtzuwachs und dem Vorbildcharakter des ,sozialistischen Lagers" wachsen.

Die von der DKP propagierte, antimonopolistische Demokratie" kennt ebenfalls zahlreiche Parallelen. Die indische KP kennzeichnet z. B. ihr analoges Konzept der ,Nationalen Demokratie“ damit, daß in ihr ,das Proletariat die Macht mit der nationalen Bourgeoisie teilt" (199). Ironischerweise ließe sich die Strategie des ,Zurückdrängens der Monopole " und der Errichtung der ,antimonopolistischen Demokratie $^{66}$ auch mit Doppelherrschaft umschreiben. Die zentralen Thesen der Stamokap-Theorie, nämlich die Verschmelzung von Staat und Monopolen, das Etappen-Modell der ,antimonopolistischen Demokratie ${ }^{66}$, die Reduktion des Klassenkampfes auf ein Problem der Umverteilung und der Funktionalisierung des Staates gegen die Monopole - diese vier Kernpunkte kennzeichnen eine Strategie, der es letztlich darum geht, den Übergang zum Sozialismus durch Auswechslung der Eliten, das heißt der Kapitalisten durch Arbeiterführer zu vollziehen - und das war auch die Strategie der Bernstein, Kautsky, Hilferding. Marx schrieb im „Achtzehnten Brumaire“: ,Der eigentümliche Charakter der Sozialdemokratie faßt sich dahin zusammen, daß demokratisch-republikanische Institutionen als Mittel verlangt werden, nicht um zwei Extreme, Kapital und Lohnarbeit, beide aufzuheben, sondern um ihren Gegensatz abzuschwächen und in Harmonie zu verwandeln. ${ }^{66}(200)$

39) G. Adhikari, The Problem of the Non-Capitalist Path of Development of India and the State of National Democracy. World Marxist Review, Vol. III, Nr. 11 (Nov. 1964).

-00) Marx, Der Achtzehnte Brumaire des Louis Bonaparte, a. a. O., S. 141. 


\section{Schlußbemerkungen}

Es ist nicht der wesentliche Zweck dieser Anmerkungen, die DKP-Agrarpolitik als solche zu kritisieren, vielmehr muß es vor allem darum gehen, mittels solcher Auseinandersetzungen die vielfältigen Probleme der Bauernfrage klarer zu analysieren, und zwar mit dem Ziel, die politische Arbeit unter den Bauern realistischer, effektiver und für die Betroffenen nützlicher zu machen. In der Praxis, draußen auf dem Land, muß sich zeigen, welche theoretischen Grundlagen mit dazu beitragen können, die rechten Ideen in den Köpfen der Menschen aufkommen zu lassen. Die theoretische Diskussion kann im besten Fall zur Erarbeitung der revolutionären Theorie in ihren die Bauern in der BRD betreffenden Teilen beitragen.

Schon heute kann jedoch resümiert werden:

„Es ist zwar unerläßlich, die Front der Kämpfe zu verbreitern, um den Kapitalismus zu schwächen und dann zu zerschlagen und die Einführung des Sozialismus vorzubereiten, aber diese Aufgabe läßt sich nicht in Zusammenarbeit mit jeder beliebigen, antimonopolistischen' Schicht der Bevölkerung eriullen. Der Klassenkampf bildet die Achse dieses Kampfes und ist also Voraussetzung für jedes Bündnis." (201)

201) Lambert, a. a. O., S. 95. 